\title{
II. Der Gegensatz von wirtschaftlichem Wieder- aufbau und Sicherheit vor Deutschland in der britischen Diskussion bis zur Kriegswende 1941
}

\section{Die konzeptionelle Herausforderung Großbritanniens durch Deutschland}

Wie der Rückgriff auf die Entwicklung der Zwischenkriegszeit zeigte, hatte sich Keynes' Aufmerksamkeit kurz vor Ausbruch des Krieges erneut dem Einfluß der Außenfaktoren auf das innere sozio-ökonomische Gleichgewicht zugewendet. Der Zusammenhang zwischen beiden, der das spezifische Antriebsmoment der Appeasementpolitik ausgemacht hatte, stellte sich nach Ausbruch der Kriegshandlungen als Doppelproblem dar: Wie würde Großbritannien erstens die zur Kriegführung notwendigen Mittel aufbringen, und welche Veränderungen in der Wirtschaftspolitik waren notwendig, um sie kriegswirtschaftlich möglichst effizient einzusetzen? In welcher Form sollte zweitens die konzeptionelle Herausforderung der NS-Wirtschaftspolitik angenommen werden? Diese Aspekte berührten in direkter Weise Großbritanniens wirtschaftliche Stellung nach Kriegsende und zugleich die Frage der zukünftigen Behandlung Deutschlands innerhalb eines durch die Ereignisse des Weltkriegs erheblich veränderten, aber noch bislang ungeformten internationalen Rahmens.

Auch Keynes' Stellung als Wirtschaftsberater hatte sich bei Kriegsausbruch nicht unwesentlich verändert. Die Publikation der „General Theory“, die ihn als prominentesten und einflußreichsten Ökonomen seiner Zeit ausgewiesen hatte, war zeitlich mit dem Beginn der Aufrüstungsdebatte zusammengefallen und hatte ihn wieder enger an die wirtschaftspolitischen Schalthebel Whitehalls herangebracht. Der Zusammenbruch Frankreichs im Frühjahr 1940 und der Schock, den der überstürzte Rückzug der britischen Truppen aus Dünkirchen auslöste, bewirkten schließlich eine politische Krise im Inneren, an deren Ende die Ablösung Chamberlains als Premierminister durch Churchill stand. Diese Krise trug entscheidend dazu bei, Keynes wieder an den Entscheidungsprozeß heranzuführen. Die personelle und organisatorische Umgestaltung der Regierung, deren markanteste Ausprägung die Einrichtung eines Kriegskabinetts war, verschaffte schließlich seinen Plänen auch innerhalb der Beamtenschaft sowie auf Ministerebene mehr Aufmerksamkeit; er selbst wurde „inoffizieller Berater“ des neuen Schatzkanzlers Sir Kingsley Wood und Mitglied in dessen neugegründetem Beraterstab ${ }^{1}$. Dabei gab ihm der Umstand, daß er im Gegensatz zur stärkeren Einbindung im Ersten Weltkrieg nun keine feste Stellung innerhalb des Beamtenapparats bekleidete, die Möglichkeit zu grö-

\footnotetext{
${ }^{1}$ Wie Keynes am 28.6.1940 an seine Mutter schrieb, werde dies den Vorteil haben, „of giving me direct access to the Chancellor of the Exchequer with any bright ideas I may have". JMK XXII, S. 190. Zu Keynes' Einfluß auf Kingsley Wood sowie zur Stellung des Schatzkanzlers vgl. den Tagebucheintrag Hugh Daltons über ein Gespräch mit Wood: „He [Kingsley Wood] says that Keynes and [Hubert] Henderson [Professor für Politische Ökonomie in Oxford und von 1939. 1944 Wirtschaftsberater im Schatzamt] and others are always, running in' to him and saying, Why don't you do so and so?' [...] I hear that they say at the Treasury, You will never do so much with that little fool'." BLPES, Dalton Diaries I/27, fol. 30 (Eintrag 19.8.1942).
} 
Berer intellektueller Formulierungsfreiheit. Dadurch gelang es ihm, eine breite Beratertätigkeit zu entwickeln, die in ihrer Wirkung nicht auf die Beamten des Schatzamts beschränkt blieb, sondern auch soziale Gruppen im Inneren, allen voran die Gewerkschaften, und bedeutende auswärtige Diplomaten und Politiker wie den US-Präsidenten Roosevelt und die Mitarbeiter der amerikanischen Botschaft umfaßte. Die Bedeutung seines Status als „demi-semi-official“2 lag darin, wie einer seiner Biographen notierte, „that, very much more than in the 1930 s, Keynes was a political' economist, whose influence, although it never equalled the brilliance of his pen, played a role unique in the annals of modern economic policy" 3 . Keynes ging es bei seiner erneuten Tätigkeit im Schatzamt darum, den Praxisbezug seiner Theorie unter Beweis zu stellen, ihn anhand der gewonnenen Erfahrungen zu überprüfen und wenn notwendig zu modifizieren. War dies schon für seine Rundfunk- und Zeitungsbeiträge zur Frage der Kriegsfinanzierung kennzeichnend, so trifft dies in noch stärkerem Maße auf die Nachkriegsplanungen zu. Als Voraussetzung hierfür betrachtete er einmal seine Parteilosigkeit, die ihm eine kaum zu überschätzende Unabhängigkeit sicherte ${ }^{4}$. Daneben wählte er aber bewußt eine Tätigkeit innerhalb der Regierung und verzichtete jetzt völlig auf die universitäre Lehre und Forschung. Jeder an ihn herangetragene Wunsch, seine Vorlesungen wieder aufzunehmen, wurde zurückgewiesen, obwohl Keynes sich der Möglichkeiten nur zu bewußt war, seine Theorie durch eine breite Lehrtätigkeit zu festigen und weiterzugeben. „But I doubt“, schrieb er zu Beginn des Jahres 1943, „if the time has yet come when one can do more from outside than from inside. I am now giving not far short of half my time to post-war questions, and there are certain projects afoot which I should be reluctant to leave to others until either they have fallen by the way or have moved somewhat nearer possible fruition. "5 Deutschlandplanungen und die Ausarbeitung der Grundlagen für eine Reorganisation der internationalen Wirtschaftsbeziehungen nach dem Krieg bildeten die miteinander verzahnten Schwerpunkte, denen sich Keynes in den nächsten Jahren widmen sollte.

Es scheint zunächst nur folgerichtig, daß Keynes in der ersten Zeit nach Kriegsausbruch sein Hauptaugenmerk auf Probleme der Kriegsfinanzierung richtete. Hatte er in der "General Theory“ das Beschäftigungsproblem noch vor dem Hintergrund stagnierender Nachfrage gesehen, die durch eine gezielte staatliche Ausgabenpolitik wiederzubeleben war, so verdeutlichte seiner Meinung nach die Diskussion über die britische Aufrüstung die Gefahren überhöhter Nachfrage. In den Ende 1939 in der "Times“ und im Frühjahr des darauffolgenden Jahres als Broschüre veröffentlichten Artikeln „How to Pay for the War" widmete er sich daher den Gefahren einer Situation exzessiver Rüstungsausgaben bei Vollbeschäftigung, in der eine erhöhte Kaufkraft bei gleichzeitig nachlassender Exporttätigkeit zu einem Ansteigen der Preise führt. Der Druck auf die Preise würde sich noch dadurch verschärfen, daß eine erhöhte Konsumbereitschaft in einer Zeit, da alle industriellen Kapazitäten auf die Produktion von Rüstungsgütern konzentriert waren, nicht auf ein entsprechend ausgeweitetes Konsumgüterangebot traf. Für Keynes bedeutete dies, daß die Kaufkraft „verschleudert" und nicht sinnvoll ge-

\footnotetext{
${ }^{2}$ Keynes an C. B. Hoover, 6.12.1945, in: JMK XXVI, S. 400 f., hier S. 400.

${ }^{3}$ Moggridge, Keynes, S. $114 \mathrm{f}$.

${ }^{4}$ Im November 1939 lehnte Keynes deshalb einen Sitz im Parlament ab. Vgl. KCKP, L/39/124-141.

${ }^{5}$ KCKP, L/43/6-8, Keynes an T. J. Carlyle Gifford, 25.1.1943.
} 
nutzt werden konnte. Zudem wies er darauf hin, daß die Verteuerung zu sozialen Härten bei denen führen würde, deren Einkommen nicht Schritt halten konnte ${ }^{6}$. Indem Keynes den analytischen Rahmen der "General Theory" anwandte, durchbrach er die geltende Politik, die auf der Annahme beruhte, daß zur Kriegsfinanzierung nur die Mehreinnahmen benutzt werden konnten, die der Steuerzahler bereit war aufzuwenden ${ }^{7}$. Diese Politik wurde von Keynes vehement attackiert, da sie kaum in der Lage war, die notwendigen finanziellen Ressourcen bereitzustellen. Die dadurch notwendige staatliche Kreditaufnahme würde zusammen mit einem kriegsbedingten Anstieg der Löhne vielmehr eine gefährliche inflationäre Spirale in Gang setzen ${ }^{8}$. Keynes forderte deshalb eine auf diese Lage abgestimmte Haushaltspolitik und schlug vor, einen Teil der Löhne zurückzuhalten, um ihn nach dem Krieg, wenn die Produktionskapazität die Nachfrage wieder überschritt, als Anschub zu nutzen ("deferred pay“). Eine genaue Kalkulation der Wirtschaftskraft des Landes sollte zunächst ermitteln, wie groß das über Steuern abschöpfbare Volumen war; daran anknüpfend sollten zum einen direkte Steuern, zum anderen Lohnabschöpfungen in der Form „erzwungener" Spareinlagen für eine allgemeine Erhöhung des Steueraufkommens sorgen und eine Dämpfung der Nachfrage einleiten; gleichzeitig ermöglichten diese finanzpolitischen Maßnahmen den Transfer wichtiger Ressourcen zur Rüstungsproduktion. Von dem Versprechen, diese „Zwangseinlagen“ nach Kriegsende wieder zurückzuzahlen, versprach sich Keynes ferner die Grundlage für eine Art staatliches Investitionsprogramm, das zur Überwindung eines antizipierten "Slump“ die zurückgehaltene Kaufkraft gezielt zur Nachfragestimulierung würde freisetzen können. Mit Blick auf die Nachkriegssituation hatte dieses Vorgehen seiner Meinung nach auch den Vorteil, daß im wesentlichen die Bevölkerung als „Schuldner“ des Staates auftreten würde und nicht die Banken. Dies gab der Regierung zum einen ein wichtiges Instrument der Einflußnahme auf die Nachfragegestaltung nach dem Krieg an die Hand; zum anderen sorgte dieses Vorgehen für eine gerechtere Umverteilung des Vermögens ${ }^{9}$. Dem entsprach seine Forderung nach einem Haushalt, der die Erfordernisse der Kriegsfinanzierung mit dem Interesse an einer anti-inflationären und sozial gerechten Lohnpolitik verband. Diese neue Form der Haushaltspolitik umriß Keynes im Frühjahr 1940 in seiner Kritik des noch von Schatzkanzler Sir John Simon vorgelegten Haushalts: „The importance of a war Budget is not because it will ,finance' the war. [...] Its importance is social: to prevent the social evils of inflation now and later; to do this in a way which satisfies the popular sense of social justice; whilst maintaining adequate incentives to work and economy." 10

Keynes' Vorstellungen über erhöhte Besteuerung und „deferred pay “ wurden verständlicherweise von den Konservativen als unglaubwürdig und von Labour sowie den Gewerkschaften als zu drakonisch zunächst zurückgewiesen. Auch die Treasury-Beam-

\footnotetext{
${ }^{6} \mathrm{KCKP}, \mathrm{BR} / 4 / 6-14$, The Economic War: What it means to us, BBC Broadcast, 11.3.1940.

7 Vgl. dazu Peden, A Matter of Timing, S. $19 \mathrm{f}$.

${ }^{8}$ Diesen Standpunkt hatte Keynes bereits in der Aufrüstungsdebatte eingenommen. Vgl. Peden, Keynes, the Economics of Rearmament and Appeasement.

9John Maynard Keynes, „Paying for the War", (The Times, 14. und 15.11.1939), in: JMK XXII, S. 41-51. Vgl. Harrod, Keynes, S. 488-494, hier S. 492; Moggridge, Maynard Keynes, S. 627-634, besonders S. 628.

${ }^{10}$ „Notes on the Budget I“, 21.9.1940, in: JMK XXII, S. 218-222, Zitat S. 218 (Hervorhebung im Original).
} 
ten begegneten der Umkehrung ihrer traditionellen Methode, welche die wirtschaftspolitische Entscheidungsfindung primär an den Erfordernissen und der Bereitschaft von Unternehmern und Steuerzahlern ausrichtete, mit kühler Zurückhaltung ${ }^{11}$. Keynes selbst sah sich dem Vorwurf ausgesetzt, sein Plan sei nur eine andere Form des „Hitlerismus"12. Dem begegnete er mit dem Hinweis, daß das Vertrauen in die Möglichkeiten steuerlicher Kriegsfinanzierung und den Sparwillen der Bevölkerung weder ausreichende Mittel zur Kriegführung garantierte noch ein probates Mittel zur Eindämmung der Inflationsgefahr darstellte. Ferner verteidigte er in einem Radiovortrag für die BBC seinen Plan, indem er die Unterschiede zur nationalsozialistischen Finanzpolitik herausstellte: „The German method is to limit expenditure by rationing a wide range of articles of general consumption. If the amount of private consumption is strictly limited in this way, it is easy enough to fix prices. But in England there are only a few articles of consumption of which we are short. So long as this is the case, a general restriction of consumption is not necessary and therefore not advisable. " Der Grund dafür, daß auch in Großbritannien Rationierungsvorschriften bestünden, sei deshalb ein gänzlich anderer, nämlich die Nachfrage nach knappen Gütern wie Butter und Schinken abzuziehen. "Thus the task of effecting a large reduction in the standard of life of the average worker is not our problem. For that purpose the German method is probably the only efficient means. We admire the skill with which it has been carried out. But our problem is a different one, namely, to prevent the better conditions of employment, the overtime earnings and the higher wages, which now prevail, from resulting in a pressure to buy more than in peace-time. For we cannot afford that in time of war." Keynes führte weiter aus, daß die wirtschaftliche Stärke Großbritanniens im ersten Kriegsjahr noch nicht angetastet worden sei und die Entbehrungen erst bevorstünden. Demgegenüber zeigten sich in Deutschland bereits die Folgen einer schon fünf Jahre währenden wirtschaftlichen Kraftanstrengung. "It is the German habit to plan carefully in advance with a patient skill in detail of which we seem incapable; and then to throw everything in without reserve. It is our habit to start with a slowness until it seems to the outsider to be too late, and to develop at long last a gradually overwhelming momentum. These same generalizations about the characters of the two adversaries apply also in the economic and financial fields. " 13

Weitere wichtige Rahmenbedingungen für die Deutschlandplanungen wurden auch durch die in gedanklicher Auseinandersetzung mit Keynes ${ }^{14}$ entwickelten Aktivitäten William Beveridges geschaffen. Beveridge, als Ökonom wie Keynes während des Krie-

${ }^{11}$ Für Hintergrundmaterial vgl. JMK XXII, S. 74-144; Moggridge, Keynes, S. 117-120; ders., Maynard Keynes, S. 633 f.; Peden, Keynes, the Treasury and British Economic Policy, S. 41 f.; Hancock/Gowing, S. 47-49.

$12 \mathrm{KCKP}, \mathrm{BR} / 4 / 6-14$, The Economic War: What it means to us, BBC Broadcast, 11.3.1940.

${ }^{13}$ KCKP, BR/4/59-63, British Finances after a Year of War, BBC Broadcast, 13. und 14.9.1940 (Hervorhebung im Original). Vgl. dazu auch die Einschätzung der nationalsozialistischen Wirtschaftspolitik durch das Foreign Office im Jahr 1939 in: Peden, A Matter of Timing, S. 18 f. Am 18. September 1940 kommentierte die Frankfurter Zeitung Keynes' Radiosendung, deren Optimismus mehr als „patriotische Leistung" denn als faktisch richtig zu werten sei: „England pflege erst langsam in Schwung zu kommen, meinte Professor Keynes, viele Leute meinten, zu langsam. Die Welt hat sich dieser Meinung schon angeschlossen, und Deutschland wird dafür sorgen, daß dem ,zu langsam' das ,zu spät‘ hinzugefügt werde." Vgl. den Presseausschnitt in KCKP, BR/4/87.

14 Vgl. Moggridge, Maynard Keynes, S. 628, 706. 
ges in Staatsdiensten und um Einflußnahme auf die kriegswirtschaftliche Entwicklung bemüht, kritisierte im Juli 1940 in scharfer Form die Kriegführung Großbritanniens ${ }^{15}$. Wie bei Keynes richteten sich seine Einwände namentlich gegen eine nur zögerliche Umstellung auf die Erfordernisse einer Kriegswirtschaft und ein Beharren auf privatwirtschaftlicher Initiative für Kriegsproduktion und Kriegsfinanzierung. Dem entsprach ein seiner Meinung nach eklatanter Mangel an Wirtschaftsplanung mit einem festen organisatorischen Platz innerhalb des Entscheidungsprozesses sowie eine völlig unzureichende Zusammenarbeit der Ministerien. Als Ergebnis stellte Beveridge eine fehlende Handlungsbereitschaft auf der Entscheidungsebene fest, die in einem grotesken Gegensatz zu den in zahlreichen Komitees und Organisationen durchgeführten Planungen stünde, deren Arbeit kritisch beargwöhnt werde. "The Germans marched to one victory through the gap between the French and British armies", bilanzierte Beveridge. "They may march to another victory through the gap between British Government departments." Der Kritik an den wirtschaftspolitischen und organisatorischen Mängeln der britischen Kriegführung entsprach es, daß Beveridge schließlich das Fehlen adäquater Kriegsziele und konkreter Friedensvorstellungen ankreidete. Nicht ohne Sarkasmus vermerkte er, daß es nicht ausreiche, auf dem Überleben des Commonwealth als einzigem nennenswerten Kriegsziel zu bestehen. Demgegenüber machte er auf die Notwendigkeit aufmerksam, einen (auch propagandistisch wirksamen) Gegenentwurf als Alternative zu einer Welt nach einem deutschen Sieg zu schaffen. Dieser Gegenentwurf sollte sich erstens von dem Grundsatz leiten lassen, die Streitmacht des Siegers nicht zur Erreichung wirtschaftlicher Vorteile einzusetzen, sondern allein zur Aufrechterhaltung von Gesetz und Ordnung; zweitens sollte der Einsatz der Staatsgewalt ausschließlich der Abwendung von Armut, mit anderen Worten der Schaffung sozialer Sicherheit dienen. „Our Peace Aims must be stated in bold and simple terms to catch the imagination of ourselves and of the New World. "Mit diesen Hinweisen hatte Beveridge den Ton angeschlagen, der für die kommenden Arbeiten von Keynes bestimmend sein sollte. Zwar trat auch aus Beveridges Zeilen überdeutlich der Einfluß hervor, den Keynes auf das wirtschaftspolitische Denken zu gewinnen begann ${ }^{16}$. Gleichzeitig wurde aber die enge Verknüpfung mit den Kriegszielen und Friedensvorstellungen, insbesondere mit den (fehlenden) Deutschlandplanungen deutlich sichtbar.

Nur kurze Zeit später zwang die außenpolitische Entwicklung Keynes dazu, die auBenpolitischen Aspekte des britischen Wiederaufbauprozesses erneut aufzugreifen. Den entscheidenden Impuls für die Fortführung seiner zuletzt im Zusammenhang mit Fragen der Kriegsfinanzierung geführten Auseinandersetzung mit der nationalsozialistischen Wirtschaftspolitik erhielt Keynes im Anschluß an die militärischen Niederlagen im Sommer $1940^{17}$ und an seine Rückkehr in die Schaltzentrale des Schatzamts. Zu diesem Zeitpunkt verstärkten sich die Hinweise aus Deutschland, die auf die Etablierung einer „Neuordnung“ hindeuteten, in deren Mittelpunkt die Reorganisation des kontinentaleuropäischen Wirtschaftsgroßraums unter nationalsozialistischer Führung stand. Im August 1940 informierte der britische Geschäftsträger in Athen das Foreign Office

${ }^{15} \mathrm{KCKP}, \mathrm{W} / 1 / 54-58$, Memorandum Beveridge, ,What is wrong with the conduct of this war in Britain“, Juli 1940. Dort auch die folgenden Zitate.

${ }^{16}$ Dafür stehen beispielhaft die Schaffung der Economic Section und die Entstehung des BeveridgeReports; vgl. Kap. III.1, besonders Anm. 24, und Kap. III.3, besonders Anm. 89.

17 Zum Epochencharakter des Jahres 1940 für die britische Politik vgl. Reynolds, 1940, S. 325-350. 
(FO) über Pläne Berlins zur Schaffung eines solchen Großraums und einer entsprechenden deutschen Hegemonialstellung, die ihm von neutralen Quellen in der Reichshauptstadt sowie aus Kreisen der griechischen Regierung zugeleitet worden waren. Nach Informationen des britischen Außenministeriums existierte in Berlin ein ausgearbeiteter Entwurf für die Schaffung einer „Paneuropäischen Föderation“. Er sah die vollständige Kontrolle über den afrikanischen Kontinent sowie Teile Osteuropas entlang einer Linie Leningrad-Kiew-Odessa vor; die Sowjetunion blieb als "asiatische Macht“ in diesem Konzept unberücksichtigt, sollte aber machtpolitisch handlungsunfähig gehalten werden. Wirtschaftspolitisch zielte der Plan auf eine handels- und währungspolitische Kontrolle der beteiligten Länder sowie auf eine Koordination der Industrien, die zu einem wesentlichen Teil unter deutsche Führung gebracht werden sollten ${ }^{18}$.

Als schließlich die britische Botschaft in der Schweiz zwei Monate später in einer Reihe von Telegrammen von der zunehmenden Besorgnis der neutralen Länder über das Stillschweigen Londons hinsichtlich seiner Kriegsziele berichtete, sah sich das Außenministerium zum Handeln gezwungen. Die weit über die bisherigen handelspolitischen Ziele der Nationalsozialisten hinausgehenden Versprechungen eines Wirtschafts- und Finanzparadieses im Rahmen der „Neuordnung“, so alarmierten die Diplomaten aus Bern, würden auf fruchtbaren Boden fallen, wenn die britische Regierung nicht mit einem Gegenkonzept antworte. Die Beamten des Foreign Office setzten daraufhin ihre Kollegen von der Treasury, dem Ministry of Information und dem Ministry of Economic Warfare (MEW) von dieser Entwicklung in Kenntnis ${ }^{19}$. Der hinter den nationalsozialistischen Plänen stehende Grundgedanke, einen stark kartellisierten und auf Autarkie zielenden Wirtschaftsblock zu schaffen, der den kontrollierten Handel mit den wirtschaftlich abhängigen Kleinstaaten an der Blockperipherie ermöglichen und ein stabiles Preis- und Marktgefüge vor allem für Agrarprodukte bieten sollte, erschien aus der Sicht Londons vor allem für die Länder Südosteuropas in gefährlichem Maße verlockend. Die Telegramme aus Bern bestätigten London die bereits durch die Unterzeichnung des Achsenpaktes durch Ungarn, Rumänien und die Tschechoslowakei geweckte Befürchtung, diese Staaten könnten in einer Art Dominoeffekt der Anziehungskraft der deutschen Wirtschaftspropaganda erliegen ${ }^{20}$. Eine rasche Gegenoffensive, welche die einem solchen Block inhärenten Abhängigkeitsbeziehungen und die Gefahr einer deutschen Wirtschaftshegemonie hervorhob, zugleich aber als Ausgangspunkt für erste Kriegszielplanungen dienen konnte, war demnach äußerst dringlich.

Dies wurde auch in einem Leitartikel deutlich, der Ende November $1940 \mathrm{im}$ „Economist“ erschien. Hitlers „Neuordnung“, so hieß es darin, ,is vile economics, but it is, perhaps, good propaganda, and we cannot be content merely to point out its falla-

${ }^{18} \mathrm{~T}$ 160/1053/F 17402, Makins (Foreign Office) an Ministry of Economic Warfare, 26.8.1940; Makins an Playfair (Treasury), 20.10.1940.

19 T 160/1053/17402/01, Halifax an Kingsley Wood, 19.11.1940; Telegramme Bern an Foreign Office, 26.9.1940, 5.10.1940, 21.10.1940, 22.10.1940, 6.11.1940, 13.11.1940.

20 Zum Hintergrund vgl. Guillebaud, der Hitlers "Neuordnung“ als Variante eines von einem deutsch-britischen Handelsgegensatz seit Beginn des Jahrhunderts gespeisten MitteleuropaTraums der Deutschen wertet. Vgl. ferner Einzig, Hitler's "New Order" in Theory and Practice; ders., Hitler's „New Order“ in Europe. Vgl. allgemein Berghahn, Westdeutsche Unternehmer, S. 301-304; Petzina, Autarkiepolitik; Eichholtz, S. 162-196; Volkmann, S. 51-76. Zur nationalsozialistischen Penetration Südosteuropas vgl. Schröder, Südosteuropa. 
cies, laugh at its pretensions and take it for granted that its inner contradictions will finally bring it down. [...] True, it is difficult at this point for the Government to make a statement of war aims which, while conveying some positive policy, will yet be general enough not to impede the conduct of the war. Yet we cannot leave unanswered the German taunt that our policy is to divide and weaken Europe for our own ends, that our victory means the return of all the insecurity connected with laissez faire and all the capacity linked with international finance. Germany, as the war goes on, will have gone far towards disproving her own case, but we shall still have to prove ours." 21 Auf Vorschlag des Foreign Office wandten sich deshalb die betroffenen Ministerien an Keynes mit der Bitte um Hilfe ${ }^{22}$. Am 20. November griff das Kriegskabinett diese Initiative auf und beschloß, den Ökonomen um ein Grundsatzpapier über die Bewertung der nationalsozialistischen Wirtschaftspläne und mögliche britische Gegenmaßnahmen zu bitten. In diesem Zusammenhang war sich das Kabinett der konzeptionellen Bedeutung bewußt, die eine solche Stellungnahme auch gegenüber den Vereinigten Staaten haben mußte. Es sah sich unter erheblichem Zeitdruck, denn sollte London bis zum Ende des Jahres nicht Position in dieser Sache bezogen haben, so würde vermutlich Präsident Roosevelt in einer für Januar geplanten Rede die Initiative ergreifen, „and it would then be difficult for us to differ openly from that statement" 23 .

Gleichzeitig verlangte auch der stärker werdende Einfluß Washingtons auf die voraussichtliche Neugestaltung der Nachkriegsordnung nach einer Antwort. Wie die Parlamentsdebatte über die Rede des Königs Ende 1939 gezeigt hatte, hatten alle Parteien den sozialen und wirtschaftlichen Wandlungsprozeß, den der Krieg auslösen würde, durchaus erkannt. Besonders die Linke sah die Notwendigkeit, frühzeitig über Friedensziele und Wiederaufbau nachzudenken. So betonte etwa Clement Attlee, daß man während der Planung für den Krieg bereits für den Frieden planen müsse: „Peace aims and recon-

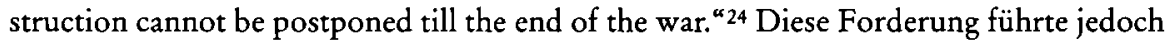
in der Ausschließlichkeit, mit der sie sich auf den Aspekt der sozialen Sicherheit im Inneren bezog, zunächst nicht zu Überlegungen über deren außenwirtschaftliche und außenpolitische Voraussetzungen, die ein Nachdenken insbesondere über mögliche deutschlandpolitische Kriegsziele impliziert hätten. Insofern widmete sich das im August 1940 geschaffene „War Aims Committee“ unter Vorsitz Attlees auschließlich Problemen der innenpolitischen Nachkriegsordnung ${ }^{25}$. Ein frühzeitiger Beginn der Nachkriegsplanungen entsprach auch kaum dem Hauptinteresse Londons, das nach dem militärischen Debakel von Dünkirchen darin liegen mußte, die USA zu einer raschen und

21 The Economist, 30.11.1940, S. 660 f., Zitat S. 661.

22 T 247/85, Harold Nicolson an Keynes, 19.11.1940. Die Meldungen der Außenstellen bestätigten im Foreign Office schon seit einigen Monaten gehegte Befürchtungen hinsichtlich einer deutschen Wirtschaftshegemonie; vgl. zum Hintergrund insgesamt Kettenacker, Krieg zur Friedenssicherung, S. 38-102.

${ }^{23}$ CAB 65/10, W.M. (40) 293rd Meeting, 20.11.1940; DzD I/1, S. 221 (Auszüge).

24355 H.C. Deb. 5s, Sp. 21, 23 (28.11.1939).

$25 \mathrm{Vgl}$. Addison, S. $122 \mathrm{f}$., $125 \mathrm{f}$. Bevin nannte am 4.10.1940 gegenüber dem Komitee als eines der wichtigsten Kriegsziele "the direction of our economy to achieve social security and the provision of a reasonable standard of living and social welfare". Ebenda, S. 125. 
dauerhaften Unterstützung der britischen Kriegsanstrengungen zu bewegen ${ }^{26}$. Churchills Hauptaugenmerk galt daher primär eher dem militärischen Tagesgeschehen und der Sicherstellung der hierfür notwendigen finanziellen und materiellen Ressourcen als der Formulierung möglicher Kriegsziele ${ }^{27}$.

Demgegenüber rückte der Erfolg von Hitlers Blitzkriegstrategie im Westen den USA die Gefahr eines antiamerikanischen Europa, dominiert von einem nationalsozialistischen Deutschland, drastisch vor Augen. Roosevelts Hoffnung, Großbritannien und Frankreich als Abwehrschild gegen die deutsche Aggression stärken zu können, war dahin und erforderte - noch vor dem eigentlichen Kriegseintritt im Dezember 1941 - ein verstärktes Engagement der amerikanischen Regierung. Hatte der Kriegsausbruch bereits die isolationistischen Strömungen innerhalb der Administration eingedämmt, so stieg der Einfluß der "Wirtschaftsinternationalisten" mit der Verschärfung der Situation an der Westfront noch weiter und überzeugte die Regierung von der Notwendigkeit, mit Überlegungen hinsichtlich einer zukünftigen Friedensordung zu beginnen, die der wirtschaftlichen Bedeutung der USA Rechnung trug. Geradezu Modellcharakter für diese Überlegungen gewann in dieser Hinsicht der britisch-amerkanische Handelsvertrag vom November 1938. Das Abkommen war ein unüberhörbares Signal für die künftige finanzpolitische Dominanz der USA. Die eigentliche politische Bedeutung des britischamerikanischen Akkords lag aber in der Signalwirkung gegenüber der nationalsozialistischen Bedrohung; daneben stellte er vor allem seitens der noch immer isolationistisch geprägten USA ein verdecktes wirtschaftliches Druckmittel im Rahmen einer Strategie des „economic containment" gegenüber Berlin dar ${ }^{28}$. Nach Kriegsausbruch und besonders nach dem Sommer 1940 entwickelte sich aus diesem deutsch-amerikanischen Gegensatz jedoch zwangsläufig auch die Frage nach einer neuen politischen und ökonomischen Nachkriegsordnung, ein Aspekt, der den konzeptionellen Einfluß der Internationalisten in der Regierung Roosevelt verstärkte. Die breite Stoßrichtung sollte nunmehr die Hegemonialpläne des Dritten Reiches genauso umfassen wie den britischen Wirtschaftsblock und das ihn stützende Währungssystem von Ottawa. Sie zielte folglich auf die langfristige Reorganisation der internationalen Wirtschaftsbeziehungen ${ }^{29}$.

Als ein erster Auswuchs dieses Klimawechsels in Washington kann Roosevelts Rede in Dayton (Ohio) am 12. Oktober 1940 gelten, in der er den Zusammenhalt der Länder Nord-, Mittel- und Südamerikas beschwor und hervorhob, zusammen werde man in der Lage sein, "to ward off any infiltration of alien political and economic ideas that would destroy our freedom and democracy ${ }^{30}$. Sechs Wochen später unterstrich Außenminister Cordell Hull die Entschlossenheit der USA zu militärischer Aufrüstung und gab die

26 Vgl. beispielhaft Churchills Mansion House-Rede am 9.11.1940, in: KCA 1940-1943, S. 4316A. Vgl. auch den Eintrag in Daltons Tagebuch: "P.M. is very optimistic about U.S. aid. They will soon, he thinks, be, in the war in fact if not in form. ' He would say to them, in effect, , if you want to watch us fighting for your liberties, you must pay for the performance. “ BLPES, Dalton Diaries $\mathrm{I} / 23$, fol. 135 (Eintrag 17.12.1940).

${ }^{27}$ Vgl. Woodward, British Foreign Policy, S. XLVII, XLIX; vgl. insgesamt Kettenacker, Krieg zur Friedenssicherung, S. 537-542.

${ }^{28}$ Junker, S. 201-224, 231 f., 241-268. Zum englisch-amerikanischen Handelsvertrag vgl. Schröder, Deutschland und die Vereinigten Staaten, S. 190-199; Schröder, Ambiguities, S. 394-398, besonders S. 396 (Zitat); Cain/Hopkins, British Imperialism 1914-1990, S. 101 f.

${ }^{29}$ Vgl. Kimball, Lend-Lease, S. 232-259; Watt, Succeeding John Bull, S. 91 et passim.

30 PPA Roosevelt, Bd. 1940, S. 460-467, Zitat S. 463. 
Einführung von Exportkontrollen bekannt ${ }^{31}$. Diese Entwicklung fand schließlich einen ersten Höhepunkt in den vom Präsidenten in seiner Jahresbotschaft vom 6. Januar 1941 dargelegten "Four Freedoms“32. Ungeachtet der militärischen Planungen sahen sich nicht zuletzt deshalb Eden und seine Beamten im Außenministerium angesichts der gezeigten Entschlossenheit der Amerikaner, den Frieden mitzubestimmen, unter Zugzwang. Der britische Außenminister stand, wie er an Churchill schrieb, unter fortwährendem Druck, „to make plain why Hitler's New Order cannot succeed and give an indication of our ability to help to meet the material needs of Europe which he is unable to supply“"33. Nachdem der ursprüngliche Wunsch, noch vor Roosevelts Rede in einer Grundsatzerklärung gegen Hitlers Neuordnungspläne Stellung zu beziehen und dadurch die konzeptionelle Initiative für die Nachkriegsordnung auf britischer Seite zu behaupten, nicht verwirklicht werden konnte, erwartete man nun, daß die Studie von Keynes nicht nur die Hegemonialpläne Deutschlands hinter den propagandistischen Äußerungen deutscher Stellen über eine „Neuordnung“ transparent machen würde; man knüpfte an sie darüber hinaus die Hoffnung, die Grundlage für eine erste öffentliche Kriegszielerklärung zu erhalten, die zugleich ein Gegenstück zu Roosevelts „Four Freedoms" darstellen sollte ${ }^{34}$.

Keynes reagierte zunächst zurückhaltend. Vielleicht erinnerte er sich daran, daß gravierende Fehlperzeptionen der wirtschaftlichen Stärke Deutschlands vor dem Krieg zu der Illusion eines „Economic Appeasement “ geführt hatten, die zugleich verknüpft war mit dem Gefühl der Überlegenheit des eigenen Wirtschaftssystems ${ }^{35}$. Deshalb vermutete er, daß auch jetzt die Vorstellungen der Ministerien seiner Auffassung nach die traditionelle Sichtweise des Schatzamtes widerspiegelten und darauf hinausliefen, der nationalsozialistischen Großraumwirtschaftsidee die Vision eines Großbritannien als Garanten des alten, auf dem Goldstandard und dem Prinzip des Laissez-faire beruhenden Systems, wie es vor 1931 bestanden hatte, entgegenzustellen. Man gehe offenbar davon aus, so ließ er Harold Nicolson vom Ministry of Economic Warfare wissen, „that we should do well to pose as champions of the pre-war economic status quo and outbid Funk ${ }^{36}$ by offering good old 1920-21 or 1930-33, i.e. gold standard or international exchange laissez-faire aggravated by heavy tariffs, unemployment, etc. etc. Is this particularly attractive or good propaganda? If you think it is, I am certainly not the man to put it across." Eine Rückkehr zum Goldstandard wie 1925 schloß er aus und ließ die Beamten wissen,

${ }^{31}$ Rede Hulls vor dem National Press Club, 26.11.1940, in: KCA 1940-1943, S. 4355A.

32 Roosevelts „Four Freedoms“ beinhalten Freiheit der Meinungsäußerung, Religionsfreiheit, Freiheit von Furcht (,a world wide reduction of armaments to such a point and in such a thorough fashion that no nation will be in a position to commit an act of physical aggression against any neighbour - anywhere in the world“) und Freiheit von Not („economic understandings which will secure to every nation a healthy peacetime life for its inhabitants“). Vgl. PPA Roosevelt 1940, S. 663-672; dt. in: KAG 1941, S. 4843C. Vgl. auch Gaddis, S. 1-31 passim; Dallek, S. 257 f.; Angermann, S. 226.

33 Public Record Office, Prime Minister's Files (im folgenden PREM) 4/100/5, Eden an Churchill, 20.5.1941. Vgl. auch Public Record Office, Foreign Office Files (im folgenden FO) 371/26532/C 6210, Memorandum Randall, „Propaganda on the ,New Order"“, ohne Datum [4.6.1941].

${ }^{34}$ CAB 65/17, W.M. (41) 8th Meeting, 20.1.1941. PREM 4/100/5, Eden an Churchill, 30.1.1941.

$35 \mathrm{Vgl}$. Peden, A Matter of Timing, S. $18 \mathrm{f}$., $26 \mathrm{f}$.

36 Angespielt ist hier auf die Rede des Außenministers Funk vom 26.7.1940, in der er sich zu den Großraumwirtschaftsplänen Berlins äußerte. Vgl. Ursachen und Folgen, Bd. 15, S. 384-391; vgl. auch Kettenacker, Krieg zur Friedenssicherung, S. 94. 
daß Wirtschaftskontrollen, vor allem währungspolitischer Art, auch über das Kriegsende hinaus fortbestehen müßten, wenn die Fehler des Versailler Friedenssystems vermieden und eine negative Rückwirkung auf Großbritanniens wirtschaftliche Entwicklung verhindert werden sollten. Das Konzept Berlins erschien Keynes vor dem Hintergrund der Erfahrungen der Krisenjahre in den zwanziger und dreißiger Jahren denn auch konsequent. Nähme man es wörtlich, schrieb er an Nicolson, so sei es „excellent und just what we ourselves ought to be thinking of doing. If it is to be attacked, the way to do it would be to cast doubt and suspicion on its bona fides. ${ }^{37}$ In begleitenden Ausführungen zu einer ersten Fassung seines Memorandums bemerkte er weiter, daß im Hinblick auf konjunkturpolitische Maßnahmen jedes britische Konzept das gleiche sei wie das von den Deutschen offerierte, „except that we shall do it better and more honestly“. Während nämlich Berlin auf die Eingliederung und Dienstbarmachung kleinerer Wirtschaftsräume, d. h. auf deren Ausbeutung ziele, biete London eine auf Zusammenarbeit unter Wahrung der wirtschaftlichen Unabhängigkeit gegründete Lösung ${ }^{38}$.

Die Grundfragen, mit denen Keynes in der Auseinandersetzung mit dem Nationalsozialismus konfrontiert wurde, treten deutlich hervor, wenn man den Artikel betrachtet, den er im November 1941 zusammen mit Edward Playfair vom Schatzamt für eine spanische Zeitung verfaßte und der bewußt als Gegenmaßnahme zur nationalsozialistischen „Neuordnung“ gedacht war. In ihm legten die Autoren die Unterschiede zwischen Sterling-Gebiet und nationalsozialistischen Großraumwirtschaftsplänen dar. Das britische Währungsgebiet greife über England und das Empire weit hinaus, so daß das Pfund Sterling flächendeckender und hinsichtlich der erwerbbaren Produkte vielseitiger einsetzbar sei als die „Funk-Mark“. Entscheidender Unterschied zu Funks „Kompensationsmechanismus" sei aber der bona fide-Charakter des Sterling-Gebiets: „It is some convenience to Germany's customers to have a bureau which will provide the financial machinery for remittance between different regions of a rationed and war ravaged Europe, cut off from overseas trade. It is a still more obvious convenience to Germany herself to have a financial dodge by which she can acquire the produce of the countries she occupies without having to pay for them in goods. But the real test between the two currencies is what each of them will buy. The Funk Mark is, in its essence, an instrument of tribute and corruption; an up-to-date variant of the exchange devices for obtaining something for nothing which Germany has worked so hard in recent years. The area pound sterling remains, in its essence, an instrument of trade which preserves to the full extent that is possible in time of war the former universality and lasting worth of the old pound sterling." Dementsprechend besäße die spanische Wirtschaft größere und vielseitigere Handelsmöglichkeiten in einem überdies vertrauenswürdigeren Sterling-Block, die keinesfalls durch die Qualität der deutschen Produkte ausgeglichen würden: „The sterling area is the largest and richest of all the territories which have a controlled economy; and the area pound is a simple mechanism to make the resources of the area available to traders all over the world. When you use it, you use the long-established and smooth, reliable machinery of the City of London, which has grown up on conducting international trade on the simplest, most honourable and cheapest basis. [...] It is not

${ }_{37}$ T 247/85, Keynes an Nicolson, 20.11.1940; JMK XXV, S. 1 f. (Hervorhebungen im Original).

38 T 245/85, Memorandum Keynes, „Proposals to Counter the German ,New Order“", 1.12.1940; KCKP, W/6/1/43-51; JMK XXV, S. 7-10; DzD I/1, S. 241-243. 
sterling itself which is useful; it is the sterling area, to which it is the key, and that is necessary to all the world. " 39

Das von Keynes skizzierte Dilemma eines deutsch-britischen Wirtschaftsgegensatzes erhellte schlaglichtartig das planerische Defizit Londons: Während einerseits eine Rückkehr zu Gold und Laissez-faire ausgeschlossen war und man gleichzeitig die Beibehaltung protektionistischer Maßnahmen als notwendig ansah, wurde andererseits jede britische Lösung entlang Keynesscher Theorien automatisch in die Nähe deutschen Blockdenkens gerückt. Diese Erkenntnis verdeutlichte für Keynes zugleich die Notwendigkeit, durch detaillierte Vorschläge eine Konzeption auszuarbeiten, welche die Möglichkeit von Wirtschaftskontrollen erlaubte und zugleich mit der Betonung internationaler Zusammenarbeit von den deutschen großraumwirtschaftlichen Ideen abrückte. Die an ihn herangetragene Bitte der Ministerien aktualisierte darüber hinaus alte Prinzipiengegensätze zwischen Keynes und der konservativen Fraktion im Schatzamt sowie der ihr nahestehenden Teil der Beamtenschaft. Was sich bereits während der Aufrüstungsdebatten abzuzeichnen begonnen hatte, wurde unmittelbar nach Bildung von Churchills Kriegskabinett, wenn auch zunächst nur in den davon stärker betroffenen Expertenkreisen, mehr und mehr zur Gewißheit: daß das Problem der ökonomischen Stabilisierung Großbritanniens und die Überwindung seiner Krisenanfälligkeit in engem Zusammenhang stand mit der Frage des künftigen wirtschaftspolitischen Kurses Londons. Es erschien immer dringlicher, unter Berücksichtigung der Versailler Erfahrungen zunächst in der Auseinandersetzung mit nationalsozialistischen Konzepten, langfristig aber auch in Konkurrenz mit den Vereinigten Staaten eine neue Grundlage für die Reorganisation des internationalen Wirtschaftsgeflechts zu schaffen. Wie sich Joan Robinson erinnerte, hatte sich im Lager der Keynesianer zu diesem Zeitpunkt bereits ein gerüttelt Maß an Niedergeschlagenheit ausgebreitet angesichts der Entwicklung im Dritten Reich, die Lloyd Georges Forderungen nach vermehrten Staatsausgaben zu bestätigen schien: „It was a joke in Germany that Hitler was planning to give employment in straightening the Crooked Line, painting the Black Forest white and putting down linoleum in the Polish Corridor. The Treasury view was that his unsound policies would soon bring him down. But the little group of Keynesians was despondent and frustrated. We were getting the theory clear at last but it was going to be too late." 40

Daß ein wirtschaftspolitisches Gegenkonzept zur nationalsozialistischen „Neuordnung" vom Außenministerium in engem Zusammenhang mit den Nachkriegsplanungen betrachtet wurde, mußte zwangsläufig, unter der Prämisse einer militärischen Niederlage Hitlers, auch die Frage nach der künftigen Behandlung Deutschlands, genauer: nach seinem ökonomischen Stellenwert innerhalb der britischen Wiederaufbaupläne und unter Berücksichtigung sicherheits- und machtpolitischer Einflußfaktoren, aufwerfen. Der Gegenentwurf, der zunächst nur als propagandistische Waffe Londons im Krieg gegen das Reich gedacht war, erwies sich aus Keynesscher Perspektive als Kristallisationspunkt wirtschafts-, sicherheits- und deutschlandpolitischer Problemlinien, deren Wurzeln bis in die Zeit des Ersten Weltkrieges zurückreichten. Es war daher auch nicht das Übergewicht des "Treasury View“, das Keynes zu einer vorsichtigen Antwort auf die Frage der

${ }^{39} \mathrm{KCKP}$, W/7/1/1-5, John Maynard Keynes/E.W. Playfair, „Que Quiere Decir El Area“, undatiert [November 1941].

${ }^{40}$ Robinson, What Has Become of the Keynesian Revolution?, S. 125. 
beiden Ministerien greifen ließ. Vielmehr schien es, als ob sich unter dem vordergründig propagandistischen Konflikt der Beginn der Auseinandersetzung um die Gestaltung der Nachkriegsordnung verbarg und schlaglichtartig den Mangel an konzeptionellen Alternativen Londons bei der Suche nach Auswegen aus der anhaltenden krisenhaften Entwicklung des Landes in der Zwischenkriegszeit bloßzulegen drohte. Wie Keynes selbst an Lionel Robbins, Wirtschaftswissenschaftler an der London School of Economics und Leiter der Economic Section of the Offices of the War Cabinet, schrieb, habe er noch nicht die geringste Vorstellung über die mögliche Ausgestaltung eines europäischen Wirtschaftssystems nach dem Krieg, weshalb er noch zögere, der Bitte nachzukommen und sich auf eine propagandistische Auseinandersetzung mit dem Nationalsozialismus einzulassen ${ }^{41}$.

Keynes erkannte jedoch, daß ein eigenständiger britischer Entwurf zur Neugestaltung der Wirtschaftsbeziehungen unumgänglich war, sollte der deutschen Propaganda ihre Anziehungskraft genommen und die Initiative in der Wirtschaftsplanung gerade auch gegenüber den USA, mit denen Verhandlungen über einen Leih-Pacht-Vertrag bevorstanden, beibehalten werden; darüber hinaus konnte aber von nicht unerheblicher Bedeutung sein, gegenüber dem Schatzamt die argumentative Oberhand zu behalten und die Deutschlandplanungen dazu zu benutzen, die Orthodoxie seiner Beamten weiter in Frage zu stellen. Die Verlängerung des Keynesschen Ansatzes auf die Deutschlandpolitik erschien gerade vor dem Hintergrund der Erfahrungen mit der Versailler Friedensordnung dringend geboten, um der Erarbeitung von Grundsätzen für ein internationales Wirtschaftsprogramm näherzukommen, das die Stabilität des Goldstandards wiederherstellen würde, ohne jedoch dessen Starrheit anzunehmen.

Wenn auch zunächst mit reichlich Skepsis arbeitend, ergriff Keynes daher die Gelegenheit und legte Mitte Januar 1941 den zuständigen Ministerien ein ausführliches Memorandum vor ${ }^{42}$. Der Kern seiner Ausführungen lag in der für ihn typischen Verbindung von binnen- und außenwirtschaftlichen mit sicherheits- und deutschlandpolitischen Aspekten. Keynes griff erneut seine Kritik an den Verantwortlichen des Versailler Friedensvertrags auf und warf ihnen vor, über ihrer Beschäftigung mit Grenzfragen und Sicherheitsgarantien den wirtschaftlichen Wiederaufbau Europas vernachlässigt zu haben. Die britische Regierung sei nun entschlossen, diesen Fehler nicht zu wiederholen. Sich auf eine Äußerung von Arbeitsminister Bevin beziehend, daß soziale Sicherheit oberstes politisches Ziel Großbritanniens nach dem Krieg sein müsse ${ }^{43}$, stellte er als

41 T 245/85, Keynes an Robbins, 28.11.1940; vgl. auch ebenda, Keynes an Nicolson, 26.11.1940.

42 T 247/85, Begleitschreiben zu Memorandum Keynes, "Proposals to Counter the German New Order“, 1.12.1940; PREM 4/100/5, „Professor Keynes' Memorandum on War Aims“, 13.1.1941; beide auch in: JMK XXV, S. 7-16.

${ }^{43}$ Bevin hatte bereits im Oktober 1940 erklärt, der Krieg werde den sozialen Konsens im Inneren derart vorantreiben, daß durch diesen Zusammenhalt sowohl Hitler geschlagen als zugleich auch der Grund für eine neue, sozial gerechtere Nachkriegsgesellschaft gelegt werde. „My war aim“, so erklärte er vor dem Bristol Rotary Club einen Monat später, ,is summed up in the phrase, ,the motive of our life must be social security'." Hitler habe das Arbeitslosenproblem durch Aufrüstung und Krieg gelöst, und die einzige Antwort hierauf bestehe darin, "that an economic order based upon construction, peace and security is being inaugurated“. Diese Bemerkung weiter erklärend, fuhr er fort: „I suggest that at the end of this war, and indeed during the war, we accept social security as the main motive of all our national life." Bevin, S. 8-15. Vgl. zum Gesamtzusammenhang Bullock, Bevin, Bd. II, S. 36-52. 
Leitgedanken einer verantwortlichen Außenpolitik nach dem Krieg die Reorganisation der Wirtschaftsbeziehungen heraus, welche die Währungsprobleme sowie die Fluktuationen des Beschäftigungsmarktes und der Preisstruktur der Zwischenkriegszeit vermeide. Die Errichtung eines internationalen Währungssystems werde die britischen Märkte auf der Basis eines gleichwertigen Güteraustauschs öffnen und jedem Land einen gleichberechtigten Zugang zu den unter britischer Kontrolle oder britischem Einfluß stehenden Rohstoffen bieten; Maßnahmen im Rahmen der britischen Arbeitsmarktpolitik erschienen ihm so letztlich auch als Basis für Hilfeleistungen zur Bekämpfung des Arbeitslosenproblems in anderen Staaten. Zur Überbrückung von unmittelbar nach Kriegsende entstehenden Ressourcenengpässen faßte Keynes den Aufbau eines Europäischen Wiederaufbaufonds ins Auge, aus dem die befreiten Länder Europas dringend benötigte Kredite beziehen konnten. Keynes verschwieg freilich nicht, daß das Ziel eines wirtschaftlichen Gleichgewichts in der Übergangsperiode von einer Kriegs- zu einer Friedenswirtschaft von erheblichen Schwierigkeiten begleitet sein würde und nur unter der Voraussetzung zu erreichen war, daß der Reichtum Großbritanniens und seiner überseeischen Verbindungen an Rohstoffen fortbestand und der Zugriff auf das Wirtschaftspotential der Vereinigten Staaten gesichert werden konnte. Er gehe auch davon aus, schrieb er dazu erläuternd in einer Begleitnote, daß die Rückkehr zur Politik des Laissez-faire, die den internationalen Handelsverkehr auf die Basis von Gold oder einer gleichwertigen Leitwährung stelle, ausgeschlossen sei. Dies entspreche nur dem gesunden Menschenverstand, würden doch die britischen Goldvorräte nach dem Krieg fast völlig aufgebraucht sein und das Land darüber hinaus mit umfangreichen SterlingSchulden konfrontiert werden. „The virtue of free trade“, so fügte er mit Bezug auf die Defekte des Versailler Systems und die in ihrer Folge herausgebildete nationalsozialistische Wirtschaftspolitik hinzu, „depends on international trade being carried on by means of what is, in effect, barter. After the last war laissez-faire in foreign exchange led to chaos. Tariffs offer no escape from this. But in Germany Schacht and Funk were led by force of necessity to evolve something better. In practice they have used their new system to the detriment of their neighbours. But the underlying idea is sound and good." 44 Auch das Schatzamt und die Bank von England hätten zwischenzeitlich ein Währungssystem aufgebaut, das sich an die positiven Aspekte der deutschen Erfahrung anlehne und das es zu übernehmen gelte, wolle man die internationalen Handelsbeziehungen nicht erneut ins Chaos stürzen. Gleichzeitig diene es aber auch dazu, die verarmten Länder Europas zu schützen, und beuge einer Wiederholung der Entwicklung nach dem Ersten Weltkrieg vor.

Erst vor dem Hintergrund dieser potentiell instabilen Konstellation wird verständlich, warum Keynes in seinem Memorandum einem besiegten Deutschland einen wichtigen und, wie er betonte, gleichberechtigten Platz in seinem Konzept zuerkannte. Deutschland werde von der Errichtung des Wiederaufbaufonds profitieren und unter geeigneten Bedingungen „and after due priority to our friends and allies“ einen entsprechenden Anteil an den vorhandenen Nahrungsmittel- und Rohstoffvorräten erhalten: „It would be senseless on our part to suppose that her [Germany's] neighbours can develop an ordered, a prosperous, or a secure life with a Germany, economically ruined, in

44 T 247/85, Begleitschreiben zu Memorandum Keynes, „Proposals to Counter the German New Order“, 1.12.1940; JMK XXV, S. 8 f. (Hervorhebungen im Original) 
their midst. Germany must be expected and allowed to assume the measure of economic recovery which flows naturally from her own qualifications and her geographical position. Germany is the worst master the world has yet known. But on terms of equality, she can be an efficient colleague." Zwar wollte Keynes seine deutschlandpolitischen Vorstellungen durch von ihm allerdings nicht näher bezeichnete "politische und militärische Friedensbedingungen“ abgesichert sehen. Doch hielt er, an traditionelle Aspekte der Friedenssicherung in der britischen Außenpolitik anknüpfend, abschließend fest: „The lasting settlement and internal peace of the continent as a whole is the only thing which suits us. It is the ultimate source of our strength in the secular European conflicts in which we have played a part that at the bottom of his heart every participant knows this." 45

$\mathrm{Zu}$ Recht hat man angemerkt, daß die Bedeutung von Keynes' Ausführungen nicht aus den Konkreta, sondern aus den seinen Überlegungen zugrunde liegenden Annahmen hervorgeht ${ }^{46}$. Das Besondere an seinen Darlegungen war, daß er die Mittel zur Realisierung einer stabilen und dauerhaften Friedensordnung wirtschaftlich definierte und Sicherheit vor einem Wiederaufleben des deutschen Militarismus durch eine Modifikation der traditionellen Politik des "Economic Appeasement" in Form einer gleichberechtigten Eingliederung Deutschlands in eine im Detail noch auszuarbeitende Neuordnung der europäischen Wirtschaftsbeziehungen erreichen wollte. „I have had to shirk the more fundamental problems“, schrieb Keynes an Robbins, "simply because I do not know what the answer is, and all I have found myself able to draft are proposals for the immediate post-war situation. These are on lines of great liberality and appeasement and go beyond anything that H.M.G. has committed itself to at present." 47 Wie weit er dabei zu gehen bereit war, ließ er die Minister in einem Begleitschreiben wissen: Deutschland unter neuer Führung "will be allowed to resume that measure of economic leadership in Central Europe which flows naturally from her qualifications and geographical position“. Der wirtschaftliche Wiederaufbau des übrigen Europa könne unter dessen Ausschluß nicht gelingen; ,and an economically reconstructed Germany will necessarily resume leadership“48.

Keynes' Bemerkungen fanden zunächst die generelle Zustimmung Hugh Daltons ${ }^{49}$, wurden vor allem aber im Außenministerium positiv aufgenommen. Dort erkannte man ihren Wert als Basis für eine geplante Erklärung Anthony Edens zu Hitlers „Neuordnung “. Die von Keynes’ Eloquenz und Sachverstand beindruckten Beamten bewerteten

45 PREM 4/100/5, „Professor Keynes' Memorandum on War Aims“, 13.1.1941; JMK XXV, S. 15 f. Das Memorandum lag Anfang Dezember 1940 in einer ersten Fassung vor und wurde als Ergebnis von Gesprächen mit dem Außenministerium am 6.1.1941 von Keynes revidiert. Vgl. T 160/ 1053/17402/01, Minute Orme Sargent, „Mr. Keynes' Draft Statement “, 6.1.1941; DzD I/1, S. 253 f. ${ }^{46}$ Moggridge, Maynard Keynes, S. 654.

47 T 247/85, Keynes an Lionel Robbins, 28.11.1940.

$48 \mathrm{~T} 447 / 85$, Begleitschreiben zu Memorandum Keynes, „Proposals to Counter the German New Order“, 1.12.1940; JMK XXV, S. 9 (Hervorhebung im Original). Für eine ähnliche aber allgemeinere Ausbreitung dieses Grundgedankens vgl. den Aufsatz von Guillebaud. Wie Keynes an den amerikanischen Wirtschaftsjournalisten Paul Einzig schrieb, stimme er mit der Schlußfolgerung dieses Artikels voll überein, „that Germany must inevitably play a prominent economic part in post-war Europe, which deserves ventilation". T 160/1053/17402/01, Keynes an Einzig, 7.1.1941.

49 Dalton bezeichnete Keynes' Memorandum als "pretty good, following the right lines, but not too precise“. FO 371/28899/W 587, Minute Dalton, 27.1.1941. 
fast einhellig seine Vorlage, in den Worten von Unterstaatssekretär Cadogan, als "the most practical and hopeful contribution to this discussion that I have seen yet" 50 . Gleichwohl verliehen sie ihren Bedenken umfassend Ausdruck. So genügte es nach Meinung Nigel Ronalds, des Leiters des General Department im Außenministerium, nicht, auf die Tatsache der wirtschaftlichen Bedeutung Deutschlands und dessen zentrale geographische Lage hinzuweisen, um daraus eine ökonomische Vorrangstellung abzuleiten. Vielmehr hing für Ronald alles von der konkreten Ausfüllung jener von Keynes nur peripher gestreiften „politischen und militärischen Bedingungen“ ab, die die Sicherheit von Deutschlands Nachbarn garantieren sollten. Darüber hinaus bemerkte er, daß der in Keynes' Ausführungen implizierte Gedanke, das Ottawa-System aufzuheben, auf erheblichen Widerstand der Dominions stoßen werde ${ }^{51}$.

Den Gegensatz von politisch-militärischer und ökonomischer Sicherheit unterstrich auch Roger Makins, der Leiter des Central Department. Er erkannte die außerordentliche deutschlandpolitische Bedeutung von Keynes' Memorandum, das nicht nur den Anstoß zur Formulierung eigener Friedensziele vor dem Hintergrund des wirtschaftlichen Wiederaufbaus und sozialer Reformen zu geben vermochte, sondern zugleich aufzeigte, $\mathrm{da}$ angesichts seiner exponierten wirtschaftlichen Stellung bereits jetzt eine Grundsatzentscheidung hinsichtlich der zukünftigen Behandlung Deutschlands gefordert war. Makins äußerte aber Zweifel, ob Keynes' ökonomischer und rekonstruktionspolitischer Primat mit der kaum zu umgehenden territorialen Neuordnung in Osteuropa zu vereinbaren war. In diesem Zusammenhang verwies er auf die polnische Kriegszielerklärung vom November 1940, die sich dezidiert für eine weitgehende wirtschaftliche Entmachtung Deutschlands und zusätzlich für Gebietsabtrennungen ausgesprochen hatte. Grundsätzlich müsse man davon ausgehen, daß im Falle eines von Keynes vorgezeichneten Wiederaufbaus Deutschland sicherlich erneut versuchen werde, politische Kontrolle zu gewinnen und die Weltherrschaft anzustreben. Bezeichnenderweise nannte er zwei in ihrer strukturellen Gegensätzlichkeit unvereinbare Möglichkeiten, die seiner Meinung nach die britische Nachkriegspolitik zu bestimmen hatten: erstens (keynesianisch) ein funktionierendes Nachkriegswirtschaftssystem, das zugleich ein ausreichendes Maß an sozialer Sicherheit für ganz Europa bot; zweitens aber das machtpolitische Zusammengehen von Großbritannien und den USA, um gemeinsam die Verantwortung für Europa sowohl in Wirtschafts- als auch in Sicherheitsfragen zu übernehmen ${ }^{52}$. Ungeachtet dieser Bedenken überwog im Foreign Office die Auffassung, daß die Arbeit von Keynes eine gute Basis für die geplante Grundsatzerklärung der britischen Regierung darstellte. Auch Eden selbst fühlte sich von dem Kerngedanken einer Trennung punitivmilitärischer und konstruktiv-wirtschaftlicher Maßnahmen angezogen. Bereits Ende Dezember 1940 hatte er nach der Lektüre einer ersten Fassung das Memorandum als „most useful stuff“ bezeichnet ${ }^{53}$.

Weitere Schützenhilfe bekam Keynes von R. W. B. „Otto “ Clarke, einem der wenigen ausgebildeten Ökonomen im Schatzamt ${ }^{54}$, der im Mai 1941 eine interne Denkschrift für das Royal Institute of International Affairs zum Thema des Aggressionspotentials der

\footnotetext{
50 FO 371/28899/W 426, Minute Cadogan, 27.12.1940; DzD I/1, S. 252.

${ }^{51}$ FO 371/28899/W 426, Minute Ronald, 11.12.1940; DzD I/1, S. 250 f.

52 FO 371/28899/W 426, Minute Makins, 3.1.1941; DzD I/1, S. $252 \mathrm{f}$.

${ }^{53}$ FO 371/28899/W 426, Minute Eden, 28.12.1940; DzD I/1, S. 252.

${ }^{54} \mathrm{Vgl}$. Clarke, Collaboration.
} 
deutschen Kriegsindustrie und der Folgen einer Repressions- oder Ausgleichspolitik Großbritanniens nach dem Krieg verfaßte ${ }^{55}$. Die deutsche Produktionskapazität, so skizzierte er das Problem, „is vitally necessary to Europe's reconstruction; the technical qualities which give Germany great military strength make her likewise the predominant supplier of the capital equipment needed for reconstruction and for progressive increase of the standard of life throughout Europe. That is the dilemma." Clarke spielte drei denkbare Alternativen für einen Friedensvertrag durch. Die Möglichkeit der vollständigen Unterdrückung, einschließlich der Zerstörung und Auflösung der Produktionskapazitäten, schloß er völlig aus: "The economic consequences of this would be tremendous delay in European reconstruction, a permanent lowering of the European standard of life and chronic instability in Europe for ever." Auch eine dauerhafte politische Kontrolle, die zwar von der Zerstörung der industriellen Kapazitäten absah, die allgemeinen Sicherheitsinteressen aber durch die Abtrennung von Teilen des deutschen Staatsgebietes und der Aufteilung des Rests in separate Staaten unter alliierter Militärverwaltung befriedigen wollte, sei kaum praktikabel. Politische Repression war nach Clarkes Ansicht nur durchsetzbar, wenn die Alliierten einen dauerhaften politischen Konsens finden würden. Die Notwendigkeit militärischer Präsenz bedeute für Großbritannien zudem eine zusätzliche wirtschaftliche Belastung und die Übernahme langfristiger Verpflichtungen auf dem europäischen Festland. Schließlich bestand noch die Möglichkeit einer Politik des Ausgleichs, die zwar die Abtrennung einiger Gebiete wie Ostpreußen und das Sudetenland vollzog, grundsätzlich aber von der Beibehaltung der deutschen Wirtschaft als einer von den Deutschen kontrollierten Einheit ausging. „After an interval of chaos, in which new governments throughout Europe were settling down, in which Britain and [the] U.S.A. would be pushing in food and raw material, and in which the Germans would be violently hated everywhere, this policy would mean German cooperation in the European system on an equal footing." Jede Nachkriegspolitik müsse, so Clarke abschließend, daher drei Ziele anzustreben versuchen: erstens die Vermeidung von Wirtschaftskrisen in Deutschland, zweitens die Umstellung von geeigneten Rüstungsbetrieben vor allem jüngeren Datums in Produktionsstätten für Konsumgüter und drittens die Garantie stärkerer Unabhängigkeit der Länder Südosteuropas durch den Ausbau des anglo-amerikanischen Handels mit diesen Ländern.

Die von Keynes und Clarke so überzeugend vorgebrachten Argumente bestimmten auch weiterhin die Diskussion in den betroffenen Ministerien. Tatsächlich beabsichtigte das Außenministerium, Keynes' Memorandum nach Vorlage im Kabinett zur Grundlage von zwei für Mai und Juli 1941 geplanten Reden Edens zu machen. Zugleich sollte in Fortsetzung traditioneller Rapprochement-Politik gegenüber den USA in Washington die Zustimmung der amerikanischen Regierung zu den Vorschlägen eingeholt werden. Auf Drängen des Foreign Office wurde Keynes angehalten, sein Konzept zu überarbeiten. Dabei sollte er es vermeiden, von einer Führungsrolle Deutschlands zu sprechen; die entsprechende Passage war durch etwas „less calculated to excite alarm than the expression ,leadership“" zu ersetzen. Daneben sollte Keynes mit dem Berater des US-Präsidenten, Harry Hopkins, bei dessen bevorstehendem Besuch in London sondieren, wie man

${ }^{55}$ FO 371/28901/W 7416, Royal Institute of International Affairs, Chatham House, „Notes on German Military Potential and the Peace Settlement“ (Memorandum by R. W. B. Clarke), 31.3.1941; DzD I/1, S. 305-308. Dort auch die folgenden Zitate. 
Washington am besten davon überzeugen könne, „that gold must be allotted a somewhat less predominant rôle, anyhow in the initial stages of economic and financial reconstruction; that the great bulk of international trade during this period must perforce be transacted on a bilateral barter-payments-agreement basis; that war time controls and exchange regulations would have to be maintained for some time after actual hostilities ceased; and that it was very much in America's interest to collaborate with us on the lines we have proposed in finding a solution of the surplus commodities problem " 56 .

Nur eine Woche später legte Keynes bereits eine neue Fassung vor, in der er im wesentlichen vermied, das Empire-Präferenzsystem zu unterlaufen. Auch war jetzt nicht mehr von einer natürlichen „wirtschaftlichen Führungsrolle“ Deutschlands die Rede; statt dessen fügte Keynes ein, dem Land müsse jenes Maß an wirtschaftlichem Wiederaufbau erlaubt werden, das seinen Fähigkeiten und seiner geographischen Lage „natürlicherweise" entspreche ${ }^{57}$. Eine weitere Überarbeitung des Entwurfs wurde Anfang Februar fertiggestellt ${ }^{58}$. Das Vorhaben, auf deren Basis eine Grundsatzerklärung des Außenministers zu erarbeiten, scheiterte zunächst freilich am Widerspruch Churchills, der Eden wenige Tage vor dessen erstem Auftritt davon in Kenntnis setzte, daß er nichts davon halte, für den Frieden zu planen, bevor man den Krieg gewonnen habe, und daß er daher die Verbreitung eines „Wirtschaftsmanifests“ zum gegenwärtigen Zeitpunkt nicht befürworten könne ${ }^{59}$.

Auch im Schatzamt hielten sich die Beamten unter Hinweis auf die Schonung der bestehenden ökonomischen Ressourcen bedeckt und warnten vor überstürzten öffentlichen Verlautbarungen über die Finanzpolitik der Nachkriegszeit, „[which might] ruin ourselves and destroy the City of London“60. Sie änderten Keynes' Passagen zum internationalen Währungssystem, strichen die Erwähnung eines Wiederaufbaufonds und ersetzten sie durch allgemeine Formulierungen, etwa den unverbindlichen Hinweis, man werde nach dem Krieg Lockerungen in der Finanzpolitik vornehmen, die eine Wiederbelebung des internationalen Handels auf der breitesten Basis ermöglichten. In den Worten eines Foreign Office-Mitarbeiters bezweckten sie damit, „to keep our hands free as regards our post-War currency policy; whereas Professor Keynes boldly comes out for an international currency system and the final abandonment of all idea of re-establishing a gold currency“"61.

Edens Rede im Mansion House vom 29. Mai 1941 war im Vergleich zu Keynes' Fassung entsprechend verändert und, wie Brendan Bracken, Churchills Parlamentarischer

56 T 160/1053/17402/01, Minute Orme Sargent, „Mr. Keynes' Draft Statement", 6.1.1941.

57 PREM 4/100/5, Memorandum Keynes, „Draft Statement to Counter the German, New Order “", 13.1.1941; vgl. DzD I/1, S. 266 f., Anm. 1-4.

58 PREM 4/100/5, „Sir Orme Sargent's Re-Draft of Mr. Keynes' Draft Statement to Counter the German ,New Order'“, 1.2.1941; DzD I/1, S. 276-280.

59 T 247/85, Eden an Churchill, 30.1.1941; Churchill an Eden, 24.5.1941; DzD 1/1, S. 275 bzw. S. $325 \mathrm{f}$.

60 FO 371/28899/W 587, Minute Orme Sargent, 16.1.1941. Zur konservativen Haltung der Londoner City in diesem Zusammenhang vgl. auch Hathaway, S. 29 f.

${ }^{61}$ FO 371/28899/W 6189, Minute Orme Sargent, 30.4.1941. Die Änderungen und weiteres Material in FO 371/28899/W 587. Im Herbst schwenkte das Schatzamt schließlich auf Keynes' Linie ein und neigte der Schaffung eines Wiederaufbaufonds unter der Bedingung der Mitarbeit der USA zu; vgl. FO 371/29015/W 12656, Treasury Memorandum, „Financial Frame-work of Post-war European Relief“, 24.10.1941. 
Privatsekretär, gegenüber dem Premierminister erklärte, „well wrapped in Foreign Office wool"62, für die allerdings auch das Schatzamt gesorgt hatte. Hitlers „Neuordnung“ - „a system of imperialist exploitation verging upon slavery“ - setzte der Außenminister das Bekenntnis zu "sozialer Sicherheit" entgegen, das er gleichermaßen als das leitende Prinzip der britischen Innen- und Außenpolitik nach dem Krieg herausstellte. Zur erfolgreichen Koordinierung der Wiederaufbaubemühungen aller Länder im Rahmen einer neu zu schaffenden weltwirtschaftlichen Ordnung sei die Zusammenarbeit des rohstoffreichen britischen Empire mit den Vereinigten Staaten unverzichtbar. Hierzu stellte Eden Lockerungen der Devisenbewirtschaftung nach dem Krieg in Aussicht. Hinsichtlich des Stellung Deutschlands in dieser Nachkriegsordnung räumte er ohne nähere Erläuterung ein, daß es „eine Rolle spielen“ müsse. Im weiteren Verlauf führte er aus, daß es nicht im Interesse Großbritanniens liege, Deutschland oder den Rest Europas nach Kriegsende „wirtschaftlich auszubeuten“. „But here“, so erklärte Eden schließlich, „I draw a firm distinction. We must never forget that Germany is the worst master Europe has yet known. [...] She must never be in a position to play that rôle again. Our political and military terms of peace will be designed to prevent a repetition of Germany's misdeeds." 63

In dieser Rede wie auch in seiner Erklärung vor der Vereinigung der Auslandspresse in London zwei Monate später erkannte der britische Außenminister zwar das Prinzip der wirtschaftlichen Rehabilitation Deutschlands prinzipiell an ${ }^{64}$. Indem er in seinen sich auf Deutschland beziehenden Bemerkungen harte Entmilitarisierungsmaßnahmen ankündigte und den Wiederaufbauaspekt nur mit dem Hinweis auf eine mögliche Eingliederung des Besiegten in die Wirtschaftsordnung nach dem Krieg streifte, erhielt die von Keynes getroffene Unterscheidung beider Elemente eine grundlegend andere Bedeutung. Obwohl Eden Passagen aus Keynes' Memorandum wörtlich übernahm, gewannen sie doch im Kontext der Betonung britisch-amerikanischer Kooperation bei der Schaffung dieser Ordnung sowie des wirtschaftlichen Führungsanspruchs beider Staaten ein nur relatives Gewicht. Edens ausführliche Bezugnahme auf Roosevelts „Four Freedoms" - und hier insbesondere die Anknüpfung des Prinzips „sozialer Sicherheit“ an die dritte Forderung nach ausreichender Befriedigung materieller Grundbedürfnisse ("freedom of want") - sowie die Verallgemeinerung von Keynes' Überlegungen zum Aufbau eines funktionierenden Wirtschaftssystems unterstrichen das primäre Interesse des Foreign Office, einen propagandistischen Gegenschlag zu Hitlers „Neuordnung " zu führen und zugleich auch die Deutschland betreffende Initiative gegenüber den USA zu behalten sowie ein gleichberechtigtes Mitspracherecht bei den Nachkriegsplanungen geltend zu machen. Mit dieser Akzentverschiebung erweckte Eden den Eindruck, daß es Ziel britischer Politik sein werde, mit militärischen Maßnahmen das Sicherheitsverlangen der Nachbarstaaten des Deutschen Reichs befriedigen, gleichzeitig aber für dessen wirtschaftlichen Wiederaufbau eintreten zu wollen, um damit einer Wiederholung des Versailler Fehlschlags vorzubeugen.

62 PREM 4/100/5, Minute Bracken, 22.5.1941; JMK XXV, S. 19.

${ }^{63}$ Für den Text der Rede Edens vgl. Eden, Freedom, S. 103-111, und DzD 1/1, S. 354-360; dt. Übersetzung in: KAG 1941, S. 5053A (mit deutschen Reaktionen).

64 Tyrell, S. 36 f. Für den Text der Rede vgl. Eden, Freedom, S. 119-123. 
Dies wich von Keynes' Konzeption insofern ab, als der Ökonom den wirtschaftlichen Außenbeziehungen Deutschlands einen eigenen sicherheitspolitischen Wert zuerkannte. Seine Überlegungen basierten auf der Idee von Sicherheit innerhalb eines nach dem Krieg aufzubauenden Wirtschaftssystems und würden daher, wie sich bald herausstellen sollte, zwangsläufig Fragen der industriellen Abrüstung und ihrer Folgen für die Rolle Deutschlands beim wirtschaftlichen Wiederaufbau Europas aufwerfen. Keynes hatte überdies mit seinem Hinweis auf den Primat der sozialen Sicherheit in der britischen Innenpolitik der Nachkriegszeit und insbesondere auf den Stellenwert einer Politik der Vollbeschäftigung die Wechselbeziehung zwischen der Behandlung Deutschlands nach der Kapitulation und der inneren sozialen und wirtschaftlichen Entwicklung Großbritanniens herausgestellt ${ }^{65}$. Die zukünftige Kontrolle Deutschlands durch dessen Eingliederung in das neu zu schaffende Wirtschaftssystem sollte dabei als wirtschafts- und sicherheitspolitisches Regulativ dienen.

Insofern stellten die in einem zentralen Punkt weit hinter Keynes zurückbleibenden Ausführungen Edens, die wenig später bezeichnenderweise auch die Zustimmung des Premierministers erhielten, zwar die erste wichtige, an die Öffentlichkeit adressierte Absichtserklärung hinsichtlich der deutschlandpolitischen Ziele Großbritanniens dar ${ }^{66}$, reflektierten jedoch auf weite Strecken noch das Interesse an einer harten Friedensregelung. Die von Keynes in diesem Zusammenhang entwickelten Überlegungen zur Währungssituation nach dem Krieg und zur Einrichtung eines Wiederaufbaufonds blieben vorerst völlig unbeachtet.

Die eher kühle Resonanz, mit der die Ideen von Keynes an höherer Stelle aufgenommen wurden, kann folglich auch nicht allein mit Churchills Desinteresse an allen nicht mit der Erringung des militärischen Sieges verbundenen Fragen erklärt werden ${ }^{67}$. Die von ihm angeschnittenen Probleme berührten Aspekte der zukünftigen wirtschaftspolitischen Orientierung Großbritanniens, die zum einen weit über die unmittelbaren militärischen Kriegsziele des Landes, zum anderen aber auch über die Deutschlandplanungen hinausgingen. Keynes selbst hatte wiederholt die Schwierigkeiten betont, denen er sich wegen des Fehlens jeglicher wirtschaftspolitischer Grundsatzentscheidungen der Regierung bei der Abfassung des Memorandums ausgesetzt sah, und auf den vorläufigen Charakter der finanz-und währungspolitischen Aspekte seiner Vorschläge hingewiesen. Er habe die Minister in seinen Überlegungen auf eine Politik festgelegt, die noch in keiner Weise akzeptiert sei, und wolle mit ihnen wirtschaftspolitische Grundsatzentscheidungen provozieren, die vor allem der Aufnahme von Wirtschaftsplanungen dienten ${ }^{68}$. An der Bereitschaft der Minister, sich mit solch weitreichenden Implikationen zu beschäftigen, schienen jedoch zumindest Zweifel angebracht.

Frederick Leith-Ross, Generaldirektor des Ministry of Economic Warfare und Chief Economic Adviser der britischen Regierung seit $1932^{69}$, sah sich jedenfalls zu einer pessi-

${ }^{65}$ In diesem Sinne wurde das Memorandum von Keynes als „the beginning of the policies of social security for all and full employment" interpretiert; Addison, S. 168.

66 Tyrell, S. 37.

${ }^{67}$ Dies gegen Tyrell, ebenda, der dementsprechend die konstruktiven Elemente in Edens Erklärungen zu positiv bewertet.

${ }_{68} \mathrm{~T}$ 247/85, Keynes an H. J. Wilson, 25.11.1940; Keynes an Nicolson, 26.11.1940; Keynes an Robbins, 28.11.1940; Keynes an R. Hopkins und H. Wilson-Smith, 15.1.1941.

${ }^{69}$ Vgl. Leith-Ross. 
mistischen Beurteilung veranlaßt. In den zwanziger Jahren hatte Leith-Ross als Berater des damaligen Schatzkanzlers Churchill wiederholt mit Keynes die Klingen gekreuzt und den orthodoxen Standpunkt des Schatzamts verteidigt ${ }^{70}$. In der Folge des Münchener Abkommens hatte Leith-Ross als Leiter der Wirtschaftsabteilung des Außenministeriums dann gegenüber Vertretern einer deutschen Wirtschaftsdelegation Mitte Oktober 1938 erklärt, die europäische Wirtschaft sei gerade gegenüber den USA in ernsthafter Gefahr, „wenn die vier Großmächte, anstatt zusammenzuarbeiten, gegeneinander arbeiten“. Die gleichartigen Schwierigkeiten der europäischen Länder und eine politische Zusammenarbeit könnten „gedeihlich nur auf der Grundlage einer gemeinsamen Wirtschaftspolitik" gelöst werden ${ }^{71}$. Leith-Ross teilte nicht Keynes' Hoffnung, daß die Lehre der Zwischenkriegszeit, nur die Schaffung und Erhaltung wirtschaftlichen Wohlstands könne dauerhaften Frieden schaffen, gelernt worden sei. „I hope indeed that this lesson has been learnt ${ }^{\text {, }}$, schrieb er am 15. Januar 1941 an Keynes, "but frankly I see little sign that our education has gone very far. Since the last war, the British workman has been getting for an equivalent amount of labour something like $50 \%$ more wages than a German, double the wages of a Pole and probably something like three times those of a Japanese workman. And goodness knows the standard of efficiency in our industries did not justify the difference. The result was a constant struggle to keep up our export trade and keep down unemployment. About ten years ago, we gave up the struggle to be competitive and used our great political and economic power to reserve markets wherever possible by means of tariffs, preferences, quotas and Trade Agreements. This policy (allied by currency adjustments) was very successful for our high cost manufacturers. It succeeded in restoring great prosperity for this country, as well as to the Empire. But it meant keeping out German exports both from the U.K. and from the Empire. It gave our people an improved standard of life but it reduced the already low standards of life in Europe. I believe that this policy helped no little to consolidate the Nazi hold on Germany and thereby contributed to the outbreak of war and that if we want a settled peace we must be prepared to modify these policies." Werde aber, so fragte Leith-Ross, die britische gegenüber einer mit niedrigeren Lohnkosten arbeitenden deutschen Industrie konkurrenzfähig sein? Bedeute die von Keynes versprochene Freiheit der Rohstoffmärkte des Empire nicht die Aufgabe des Systems der „Imperial Preferences“ für britische Hersteller, und könne sich das Land dies leisten? Wenn nun der britische Export nach dem Krieg weniger konkurrenzfähig werde, so mutmaßte er, würde eine Lockerung bestehender Schutzmaßnahmen nur das Risiko erheblicher Arbeitslosigkeit erhöhen; nach einem langen und bitter geführten Krieg werde es ohnedies schwierig sein, die öffentliche Meinung von einer wirtschaftspolitischen Kursänderung zu überzeugen, nur um Deutschland auf die Beine zu helfen ${ }^{72}$.

70 Clarke, Keynesian Revolution, S. 30f., 88-91, 117-120.

7 Vermerk über ein Gespräch zwischen Sir Frederick Leith-Ross und den Herren Rüter und v. Süsskind, 18.10.1938, in: ADAP, D, IV, S. 273-275. Vgl. den Vermerk des Leiters der Deutschen Wirtschaftsdelegation in London, 10.11.1938, sowie Botschaft London an Auswärtiges Amt, 19.12.1938, und Botschaft London an Auswärtiges Amt, 25.1.1939, in: ebenda, S. 268 f., 303 f. und $339 f$.

72 T 247/85, Leith-Ross an Keynes, 15.1.1941. Der Aspekt der mangelnden Wettbewerbsfähigkeit taucht in fast deckungsgleicher Argumentation noch 1946 in einer Einschätzung der britischen Exportindustrie durch die amerikanischen Militärs auf; vgl. Hathaway, S. 26 und S. 325, Anm. 19. 
Das Dilemma, auf das Leith-Ross hinwies, bestand darin, daß der Aufbau eines dauerhaften Friedens auf der Basis innerer und äußerer wirtschaftlicher Sicherheit, wie Keynes es in seinem Memorandum skizziert hatte, eine durchgreifende Revision der bestehenden Wirtschaftspolitik mit dem Ziel der Öffnung der britischen Märkte für die internationale Konkurrenz implizierte. Eine solche Öffnung wies freilich einen Doppelcharakter auf: Entweder half man Deutschland beim wirtschaftlichen Wiederaufstieg und gestattete ihm, wie Keynes es vorgeschlagen hatte, die Wiedergewinnung einer Position ökonomischer Stärke, für deren friedliche Ausrichtung die Integration seiner Industrien in ein multilaterales Handelssystem dann freilich eine notwendige Bedingung war. In diesem Fall würde man allerdings die britische Industrie zu einem Zeitpunkt dem Druck eines Freihandelssystems aussetzen, zu dem sie sich selbst noch schutzbedürftig und wenig konkurrenzfähig in einem Genesungsprozeß befinden würde. Um der Erreichung äußerer Sicherheit willen riskierte diese Option mithin die Sicherheit im Inneren. Oder aber London bestand auf der Fortschreibung der Ottawa-Prinzipien als Mittel zur Absicherung des eigenen Wiederaufbaus, riskierte dann aber eine Wiederholung der Autarkie- und Blockpolitik der dreißiger Jahre, die, in den Augen von Leith-Ross, ein wesentliches Antriebsmoment der nationalsozialistischen Expansion waren. Diese Option drohte also umgekehrt die äußere Sicherheit zugunsten eines Primats des inneren sozialen und wirtschaftlichen Friedens aufs Spiel zu setzen. Angesichts dieses von LeithRoss so prägnant herausgearbeiteten Strukturproblems wird deutlich, daß die deutsche Propagandaoffensive der „Neuordnung“ für Großbritannien eine kaum zu unterschätzende Auslöserfunktion besaß. Im Kern legte sie das Dilemma der britischen Außenpolitik bloß und rückte die Gefahr eines weitreichende Konsequenzen implizierenden Glaubwürdigkeitsverlusts vor Augen für den Fall, daß London das Doppelziel verfolgen sollte, wirtschaftspolitisch an den Prinzipien von Ottawa festzuhalten und gleichzeitig auf einer Einbindung des deutschen Wirtschaftspotentials zu bestehen. Wie auch immer man das Problem aber betrachtete, der ökonomische Wiederaufbau und die Sicherheit Großbritanniens hingen untrennbar von der Frage ab, welche Rolle man dem deutschen Wirtschaftspotential in einer künftigen Friedensordnung zuzuweisen bereit war.

Keynes selbst teilte Leith-Ross mit, daß er dessen Pessimismus nicht voll teile - allerdings auch nicht entkräften könne -, und versicherte dem Schatzamt, er schlage nicht die Aufgabe bilateraler Zahlungsabkommen vor, sondern vielmehr deren Übergehen in einen größeren internationalen und dauerhaften Rahmen ${ }^{73}$. Allerdings waren eine Reihe von prinzipiellen Fragen aufgeworfen, die an der Basis liebgewonnener Doktrinen rüttelten: Wie weit mußte das bestehende System modifiziert und neuen Gegebenheiten, zumal der von Leith-Ross in seinem Memorandum deutlich hervorgehobenen Bedeutung der ökonomischen Potenz der Vereinigten Staaten und ihrer Wirtschaftskonzeption, angepaßt werden? Welcher Kurs war zwischen der Neigung zu Protektionismus und Bilateralismus, die etwa im britischen Industrieverband vorherrschte ${ }^{74}$, und einer

${ }^{73}$ T 247/85, Keynes an Leith-Ross, 21.1.1941; Keynes an R. Hopkins und H. Wilson-Smith, 15.1.1941.

${ }^{74}$ Die Federation of British Industries hatte bereits vor dem Einmarsch deutscher Truppen in die Tschechoslowakei bilaterale Gespräche mit deutschen Industriellen aufgenommen und befür- 
Laissez-faire-Nostalgie, die vor allem das Schatzamt prägte, zu steuern? Und vor allem, welcher Stellenwert kam dem Faktor Deutschland aus wirtschafts- und sicherheitspolitischer Perspektive in diesem Zusammenhang zu? Was bedeutete konkret die wiederholt vorgetragene Beteuerung, aus den Fehlern von Versailles gelernt zu haben?

Damit war zugleich die Frage nach der Rolle Deutschlands in Europa prinzipiell aufgeworfen. Für das Foreign Office bestand das Hauptproblem, wie Roger Makins es pointiert formuliert hatte, in „admitting that Germany should be permitted to resume eco-

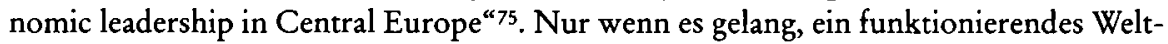
wirtschaftssystem aufzubauen und zugleich Großbritannien und die USA zu Garanten für die Sicherheit Europas zu machen, hatte Keynes' deutschlandpolitisches Stabilisierungskonzept nach Ansicht von Makins Aussicht auf Erfolg. Damit umriß er zugleich das Spannungsfeld, in dem sich die Beamten des Außenministeriums in ihrem Zwiespalt zwischen Tradition und Modernisierungszwang bewegten. Aber auch für Makins erschienen die damit verknüpften Grundsatzfragen kaum kalkulierbar, zumal sie, wie er später bemerken sollte, einen umfassenden Prozeß des Umdenkens und ein Abrücken von traditionellen politischen und wirtschaftlichen Denkweisen erforderten ${ }^{76}$.

\section{Die konzeptionelle Herausforderung Großbritanniens durch die USA}

Es waren aber nicht nur innerbritische Debatten, die Einfluß auf Edens Rede im Mansion House nahmen. In den USA hatten sich seit Bekanntwerden der Pläne für eine nationalsozialistische „Neuordnung“ die Fronten gegen Berlin weiter verhärtet". Auch die Deutschlandplanungen waren in eine neue Phase getreten. Es stand zu erwarten, daß Edens Mansion House-Rede in den USA mit großem Interesse verfolgt werden würde. Gleichzeitig war davon auszugehen, daß im Kontext der auf britischer Seite von Keynes geführten Lend-Lease-Verhandlungen dessen deutschlandpolitisches Konzept besondere Aufmerksamkeit für sich in Anspruch nehmen konnte.

In seinen Erinnerungen schreibt Eden, er habe Präsident Roosevelts Bitte, Keynes' Ideen in seiner Rede auszuklammern, bewußt zurückgewiesen ${ }^{78}$. Angesichts der Sachlage muß diese Behauptung eher bezweifelt werden. Roosevelt war vom Inhalt des Keynesschen Memorandums zunächst durch seinen Berater Harry Hopkins in Kenntnis gesetzt worden, der während seines Londoner Aufenthalts im Januar 1941 eine Kopie

wortete die Fortdauer von Import- und Exportkontrollen, in der Konsequenz also die Rückkehr zum Tauschhandel. Die London Chamber of Commerce erklärte 1942 in einer Studie über den industriellen Wiederaufbau nach dem Krieg, der internationale Handelsverkehr solle auf der Basis eines permanenten bilateralen Güteraustauschs stattfinden. Vgl. Gardner, Sterling-Dollar Diplomacy, S. 30-35, bes. S. 32. Zu Keynes' Einstellung in dieser Frage vgl. FO 371/18899/W 6635, Keynes an F. T. Ashton-Gwatkin, 25.4.1941.

${ }^{75}$ FO 371/28899/W 426, Minute Makins, 3.1.1941; DzD I/1, S. $252 \mathrm{f}$.

${ }^{76} \mathrm{FO} 371 / 29015 / \mathrm{W} 13829$, Minute Makins, 31.10.1941.

77 Zur amerikanischen Reaktion auf Hitlers "Neuordnung" und auf japanische Großraumwirtschaftspläne im pazifischen Raum vgl. Junker, S. 201-224, bes. S. 237-241.

${ }^{78}$ Eden, The Reckoning, S. $258 \mathrm{f}$. 
gesehen und Eden zufolge nahezu enthusiastisch reagiert hatte ${ }^{79}$. Hopkins gab Keynes jedoch zu verstehen, daß vor einer öffentlichen Erklärung dem US-Präsidenten eine Gelegenheit zum Studium des Memorandums gegeben werden sollte; Roosevelt sei diesbezüglich etwas empfindlich, „as he regarded the post-war settlement so to speak as being his preserve" 80 .

Ende Mai 1941 hatte Keynes dann im Verlaufe seiner Lend-Lease-Verhandlungen in Washington selbst Gelegenheit, mit dem Präsidenten seinen Entwurf zu diskutieren. Bereits zuvor hatte er vor der Presse erklärt, daß im Falle eines deutschen Sieges die amerikanische Wirtschaft zerstört würde; die lebenswichtigen Wirtschaftsbeziehungen der USA wären dann nicht nur zu Europa, sondern auch zu Lateinamerika abgeschnitten ${ }^{81}$. Das zweieinhalbstündige Treffen fand schließlich einen Tag, nachdem Roosevelt in einer Rundfunkrede den nationalen Notstand proklamiert und damit seinerseits auf die nationalsozialistische „Neuordnung“ geantwortet hatte, statt. Der Präsident hatte Hitler vor allem vor einer Penetration Lateinamerikas gewarnt und die „Einigkeit der amerikanischen Republiken“ beschworen. Die Deutschen planten, „to treat the Latin American Nations as they are now treating the Balkans. They plan then to strangle the United States of America and the Dominion of Canada. [...] Tariff walls - the Chinese walls of isolation - would be futile. Freedom to trade is essential to our economic life. We do not eat all the food we can produce; and we do not burn all the oil we can pump; we do not use all the goods we can manufacture. It would not be an Amercan wall to keep Nazi goods out; it would be a Nazi wall to keep us in. “ Roosevelt kündigte „aktiven Widerstand“ gegen jede Bedrohung dieser Hemisphäre von seiten Deutschlands an ${ }^{82}$. Vor diesem Hintergrund zeigte sich Roosevelt ablehnend gegenüber der Idee britisch-amerikanischer Friedensgespräche, ließ aber durchblicken, daß er gegen streng geheime Diskussionen, „hinter der Szene“ und ohne öffentliche Erklärungen, nichts einzuwenden habe. Er ließ Keynes weiterhin wissen, daß jede Friedensordnung auf der Entwaffnung ganz Europas basieren müsse. Die Sicherheit der europäischen Staaten sollte durch Großbritannien und die USA als „Polizisten“ garantiert werden. Er versicherte Keynes, daß die USA diesmal ihren Teil an der Verantwortung der politischen und wirtschaftlichen Nachkriegsentwicklung zu tragen bereit sein werden. Roosevelt dachte dabei analog der panamerikanischen Linie seiner Rede vom Vortag an eine Lösung, die Lateinamerika mit einschloß. Wie Hopkins bereits vermutet hatte, kritisierte der Präsident dagegen Keynes' Denkschrift wegen seiner britischen bzw. europäischen Prioritäten. Sie vermittele zu sehr den Eindruck, als habe Großbritannien die Initiative in den Nachkriegsplanungen übernommen, und sei zu sehr auf Europa konzentriert. Botschafter Halifax wies das Foreign Office zu Recht darauf hin, daß dies durchaus als Warnung gemeint war. Mit

79 FO 371/28899/W 587, Minute Eden, 20.1.1941; PREM 4/100/5, Eden an Churchill, 30.1.1941. Vgl. auch FO 371/28899/W 587, Cadogan an Keynes, 21.1.1941. Dalton bezeichnete Hopkins, mit dem er Anfang Februar zusammentraf, als „first-class scout for the President“. BLPES, Dalton Diaries I/24, fol. 27 (Eintrag 4.2.1941); vgl. ebenda, I/25, fol. 28 f. (Eintrag 24.7.1941).

${ }^{80}$ FO 371/28899/W 6189, Minute Cadogan, 30.1.1941; DzD I/1, S. 274.

${ }^{81} \mathrm{KCKP}, \mathrm{W} / 8 / 113,115$, Zeitungsausschnitte New York Times und Wall Street Journal vom 9.5.1941.

82 PPA Roosevelt 1941, S. 181-194, Zitat S. 183 f.; dt. in: KAG 1941, 5043A. Vgl. zum Hintergrund Junker, S. 258-263; Dallek, S. 265 ff. 
Blick auf die Lend-Lease-Verhandlungen, in deren Verlauf die Amerikaner auch Fragen nach dem wirtschaftspolitischen Kurs in der Nachkriegszeit aufwerfen würden, war es nach Ansicht von Halifax wenig ratsam, daß London die Initiative vor einer entsprechenden Erklärung der USA übernahm; zudem bestand die Gefahr, daß London in entsprechenden Verlautbarungen etwas preisgab, was Washington ohnehin zur Bedingung für Lend-Lease machen würde, und dadurch die eigene Verhandlungsmasse im Vorfeld beschnitt. "I am afraid“, so schloß Halifax, "that the above criticisms would not leave much substance in the paper, and both Keynes and I got the feeling that the President was not very keen on a speech being made on the lines of Keynes' paper at this time. ${ }^{\text {" } 83}$

Die Bemerkungen von Hopkins und Roosevelt gegenüber Keynes machten unverkennbar deutlich, daß die USA, obgleich bis dahin noch nicht Kriegsteilnehmer, durch eine erste vertragsrechtliche Vereinbarung über Hilfeleistungen an die Gegner der Achsenmächte zu einem der Hauptakteure auf der diplomatischen Bühne aufgestiegen waren $^{84}$ und in deutschland- wie europapolitischen Fragen nach dem Krieg eine Führungsposition beanspruchen würden. Der „Lend Lease Act“ vom 11. März 1941, von Churchill in Verkennung der eigentlichen amerikanischen Interessenlage als "the most unsordid act " bezeichnet ${ }^{85}$, markierte nicht nur den Beginn einer beispiellosen Mobilisierung der amerikanischen Exportindustrie ${ }^{86}$, mit der die USA auch nach außen ihren Willen zeigten, aktiv gegen die Diktatoren in das Geschehen einzugreifen, sondern konnte zugleich als ein Element des Open Door-Konzepts betrachtet werden. Nicht zuletzt auch die Erfahrung der deutsch-amerikanischen Wirtschaftskonkurrenz in Lateinamerika in den dreißiger Jahren hatte, wie Roosevelts "Kaminplauderei“ zeigte, die Amerikaner davon überzeugt, daß eine stabile Nachkriegsordnung eine umfassende wirtschaftliche Neuordnung voraussetzte, die durch die Beseitigung der bestehenden Wirtschaftsblöcke und der sie regelnden Prinzipien den Frieden garantieren sollte ${ }^{87}$.

Dies war auch der Inhalt einer Rede von Außenminister Cordell Hull zehn Tage vor dem Treffen zwischen dem britischen Ökonomen und dem US-Präsidenten. In ihr hatte er deutlich auf prinzipielle Berührungspunkte mit Keynes' Konzeption aufmerksam gemacht, zugleich aber mit einem Plädoyer für den uneingeschränkten Freihandel freilich auch auf einen fundamentalen Gegensatz hingewiesen. Am 18. Mai 1941 hatte Hull in einer Ansprache zur Eröffnung der Außenhandelswoche fünf Prinzipien aufgestellt, von denen seiner Auffassung nach jede Nachkriegspolitik geleitet sein müsse. Erstens dürfe

${ }^{83}$ T 160/1053/17402/01, Halifax an Foreign Office, 28.5.1941; DzD V/1, S. 330 f. Keynes an Kingsley Wood, 2.6.1941, in: JMK XXIII, S. 103-113, bes. S. 109 f.; Harrod, Keynes, S. 509 f. Zur Besorgnis innerhalb der Treasury über die bevorstehenden Verhandlungen und zur Befürchtung daraus erwachsender finanzieller Abhängigkeit von den USA vgl. BLPES, Dalton Diaries I/23, fol. 75 (Eintrag 24.10.1940).

${ }^{84}$ Duroselle, S. 973.

${ }^{85} \mathrm{Vgl}$. ausführlich Kimball, The Most Unsordid Act.

${ }^{86} \mathrm{Vgl}$. Holbo, S. $957 \mathrm{f}$.

${ }^{87}$ Zu Lateinamerika, Roosevelts "Good Neigbour Policy" und der deutsch-amerikanischen Wirtschaftskonkurrenz vgl. Williams, Tragödie, S. 187-229, bes. S. 199-211; Hull, Bd. I, S. 493-503, 601-611, 813-830; Gardner, Economic Aspects, S. 47-63, 109-132, 157-162, 194-216; Schröder, Deutschland und die Vereinigten Staaten, S. 201-261; ders., Das Dritte Reich, S. 107-152, bes. S. 137-145; Angermann, S. 200-205 et passim; Pommerin; MacDonald, S. 365-396. Allgemein: Van Alstyne, S. 584-596; Karnes, S. 730-741. Zu den wirtschaftlichen Kriegszielplanungen der US-Administration vgl. neben Gardner, Sterling-Dollar Diplomacy, auch Gaddis, S. 18-23. 
extremem Nationalismus nicht wieder erlaubt werden, eine Politik exzessiver Handelsbeschränkungen zu verfolgen; Nichtdiskriminierung müsse zweitens zur Regel der internationalen Handelsbeziehungen werden; allen Nationen müsse drittens der Zugang zu den Rohstoffmärkten ohne Benachteiligung gewährt werden; viertens sollten die den Warenfluß regelnden internationalen Abkommen so verwirklicht werden, daß die Interessen der Verbraucherländer voll geschützt blieben; und fünftens waren Institutionen für die Abwicklung des Finanzverkehrs zu schaffen, um durch Bereitstellung ausreichender Kredite die Entwicklungsfähigkeit zu sichern ${ }^{88}$.

Obwohl Keynes noch Änderungsvorschläge für die Rede Edens nach London telegraphierte, um stärker auf Hulls Ausführungen Bezug zu nehmen und den Eindruck eines britisch-amerikanischen Einvernehmens in den deutschlandpolitischen Fragen zu erwecken $^{89}$, kann es doch nicht verwundern, daß der Auftritt des britischen Außenministers von einer in ihrer Sicht noch nicht durch den Schock von Pearl Harbor getrübten amerikanischen Presse kritisiert wurde. Sie nahm vor allem Anstoß an Edens nach ihrem Empfinden widersprüchlicher Vorstellung eines militärisch harten Friedens, der gleichzeitig den wirtschaftlichen Bankrott und das Gespenst eines hungernden Deutschland ausschließen sollte. Die Journalisten hielten dagegen, daß ein Friedensplan auf der Basis von Edens Ausführungen eine Lösung der anstehenden wirtschaftlichen Probleme in einem internationalen Rahmen eher behindern würde. Im Unterschied zu Edens eigener Darstellung urteilte der British Press Service zusammenfassend, die Reaktion sei favourable but not enthusiastic “ gewesen. Von 22 Leitartikeln hätten 13 Edens Aussage, daß Deutschland nie mehr eine beherrschende Rolle übernehmen dürfe, ausdrücklich beklagt, darunter pro-britische Blätter wie die New York Times. Während die New York Herald Tribune kritisierte, Eden habe nicht genügend zwischen Hitler und Deutschland unterschieden, stellte der Washington Times-Herald die moralische Unvereinbarkeit der militärischen und wirtschaftlichen Zielsetzungen Edens heraus und sprach in diesem Zusammenhang von "some clever double-talk about the post-war world“, das der Realität der Bedeutung Deutschlands kaum entspreche ${ }^{90}$. Die breite amerikanische Kritik an dieser ersten öffentlichen Stellungnahme des britischen Außenministers zur Zukunft Deutschlands bezog sich daher nicht auf eine zu enge Anlehnung an Keynes' Konzeption und einer zu gemäßigten Linie hinsichtlich der Beseitigung des Nationalsozialismus und seiner wirtschaftlichen Grundlagen. Vielmehr war die Unterscheidung von militäri-

${ }^{88}$ Hulls Rede in: The Times, 20.5.1941; DzD V/1, S. 333-335; dt. Auszüge in: KAG 1941, S. 5034C; Wortlaut der Washington Post vom 19.5.1941 in: KCKP, W/8/123. Vgl. ferner Gardner, SterlingDollar Diplomacy, S. 4-22. Wie der damalige amerikanische Botschafter in London, Gilbert Winant, im März 1941 gegenüber Dalton bemerkte, war Hull "the next most important man after the President, and has a very wide outlook and is always balancing one possibility against another ${ }^{\text {} . ~ B L P E S, ~ D a l t o n ~ D i a r i e s ~ I / 24, ~ f o l . ~} 67$ (Eintrag 20.3.1941). Vgl, dagegen die zur gleichen Zeit geäußerte Meinung des US-Sonderbeauftragten in London, Averell Harriman: „He [Harriman] does not think much of Hull and agrees the State Department are remote and difficult." Zum Hintergrund Harriman/Abel, S. $17 \mathrm{ff} ., 27 \mathrm{ff}$.

${ }^{89}$ Material zu den Änderungen in FO 371/28899/W 6227. Vgl. auch Eden, Freedom, S. 109.

90 Kommentierte Zusammenstellung der Pressereaktionen in T 247/85, ,Eden on Peace Aims “ (Survey of American Press). Pressematerial auch in FO 371/28900/W 7131. Vgl. dagegen Eden, The Reckoning, S. 102. Diese Beurteilung steht im Gegensatz zu Keynes' eigener Einschätzung, der am 2. Juni 1941 Ronald telegraphierte, die Rede sei sehr gut aufgenommen worden. Vgl. DzD I/1, S. 360, Anm. 6. 
schen und wirtschaftlichen Maßnahmen ihres Kontexts beraubt und erschien antagonistisch und deshalb wenig realistisch". Die vagen Hinweise auf mögliche Lockerungen der Wirtschaftskontrollen nach dem Krieg mußten jedenfalls die Skepsis solcher Multilateralisten wie Außenminister Hull hervorrufen.

Bereits in diesen frühen Verlautbarungen manifestierte sich also ein Kern für mögliche Interessengegensätze zwischen London und Washington in der Außenwirtschafts- und Deutschlandpolitik. Es zeigte sich ebenfalls, daß die Ausgangspositionen beider Länder recht unterschiedlichen Charakter trugen. Im Gegensatz zu den Vereinigten Staaten, in denen sich die Prinzipien des „New Deal “ bereits recht früh auch nach außen gewendet hatten und ihre korporatistische Verwurzelung für eine über die Administrationen weit hinausgehende Durchschlagskraft sorgte, lag in Großbritannien die Ausarbeitung wirtschaftspolitischer Leitsätze allein in den Händen von Keynes und einigen wenigen Ökonomen in der Economic Section of the Cabinet wie Robbins, James Meade und „Otto“ Clarke. Über die Einsicht hinaus, daß jede Nachkriegslösung auf den Erfahrungen der frühen zwanziger und der dreißiger Jahre beruhen mußte, d. h. auf der Abkehr vom Goldstandard und dem Aufbrechen der deutschen und japanischen Blockbestrebungen bei gleichzeitigem Schutz des Sterlinggebiets, mangelte es deutlich an genaueren Vorstellungen - zum Leidwesen Keynes', der überdies, wie wir gesehen haben, auf die Abneigung der zuständigen Treasury-Minister stieß, sich auf eine bestimmte Währungs- und Finanzpolitik einzulassen. Während in Großbritannien jegliche Planungen noch von der Unwägbarkeit der alles entscheidenden Frage erschwert wurden, welche wirtschaftlichen Folgen der Krieg für das Land haben und wie sich die den machtpolitischen Spielraum wesentlich bestimmende Ressourcenlage gestalten würde, erzielten die USA durch ihre sich abzeichnende Stellung als größter Kreditgeber nach Beendigung des Krieges und den sich abzeichnenden Planungsvorsprung, der, wenngleich im Detail noch nicht präzisiert, auf einem relativ geschlossenen ökonomischen Weltbild und dessen ausgeprägter Verwurzelung in Geschäftskreisen beruhte, entscheidende Vorteile92.

\section{Britischer Wiederaufbau oder Sicherheit vor Deutschland? Die Intensivierung der Debatte im Außen- und Handelsministerium}

Auch wenn jede weitere Diskussion wegen einer anderen Prioritätensetzung Churchills, der Vorläufigkeit von Keynes' Kerngedanken und einer gewissen Abneigung in weiten Teilen der Beamtenschaft vorerst verschoben war, so bleibt an dieser Stelle doch festzuhalten, daß die Deutschlandplaner Londons in Keynes einen entscheidenden Initiator gefunden hatten. Ungeachtet der Tatsache, daß Keynes' Aktivitäten zunächst auf wenig fruchtbaren Boden gefallen waren und nur durch eine Reihe von „Filtern“ in die Grundsatzerklärungen Edens Eingang gefunden hatten, war der Zusammenhang zwischen bri-

${ }^{91}$ Dies gilt auch für Edens Rede vor Vertretern der Auslandspresse am 29.7.1941, die sich direkter mit Deutschland befaßte als die Mansion House-Rede. Vgl. Tyrell, S. 36 f. Vgl. auch T 160/1250, R.E.S. 12, Note by the Foreign Office, 29.7.1941.

92 Vgl. auch die Hinweise bei Gardner, Sterling-Dollar Diplomacy, S. 1-39; Penrose; Gaddis, S. 1823; Loth, Teilung, S. 32-37; Kolko, S. 245-248. 
tischem Wiederaufbau, der Schaffung einer Nachkriegswirtschaftsordnung und der Sicherheit gegenüber Deutschland als Problem umrissen worden. Während eine TreasuryDelegation unter Keynes' Leitung mit den Amerikanern vor diesem Hintergrund mit der vertraglichen Ausarbeitung der Lend-Lease-Lieferungen begann ${ }^{93}$, trieben interne Diskussionen auch in Whitehall das Thema voran.

Für diese Kontroverse ist ein im Juni 1941 dem Außenministerium vorliegendes Memorandum des Assistant Secretary im Handelsministerium, R. J. Shackle, über die Bedingungen des wirtschaftlichen Wiederaufbaus von besonderer Bedeutung ${ }^{94}$. Im Mittelpunkt stand der bereits von Leith-Ross hervorgehobene Gegensatz zwischen dem Freihandelsprinzip, das als notwendige Voraussetzung für die Verhinderung erneuter deutscher Hegemonialbestrebungen herausgestellt wurde, und der Fortsetzung einer handels- und währungspolitischen Blockpolitik, die für den Genesungsprozeß der britischen Wirtschaft in der unmittelbaren Nachkriegszeit als vorteilhaft angesehen wurde. Dabei drehte sich letzlich alles um die Frage, welchen wirtschaftlichen Stellenwert unter sicherheitspolitischen Zielsetzungen Deutschland im Wiederaufbauprozeß einnehmen würde: „It is the presence or absence of a monopolistic rôle for Germany which will determine whether the future economic organisation of Europe contains the seeds of political danger or not." Konnte der Wiedergewinnung einer monopolistischen Stellung und damit einem Wiederaufleben deutscher Aggressionspolitik auf dem Kontinent dadurch vorgebeugt werden, daß dem deutschen Wirtschaftspotential ein ihm entsprechender Platz in den internationalen Handelsbeziehungen zugewiesen wurde, wie es Keynes vorschwebte? Und bedeutete dies trotz des Beitrags, den Deutschland in dieser Weise zur wirtschaftlichen und politischen Stabilität Westeuropas leisten konnte, daß eine Blocklösung, die für Großbritanniens krisengeschüttelte Wirtschaft wenigstens ein Mindestmaß an Sicherheit für den eigenen Wiederaufbau gewährleistete, ausgeschlossen war? War also die Eindämmung des „Sicherheitsrisikos Deutschland" nach dem Krieg verknüpft mit der Preisgabe des Ottawa-Systems und dem Aufbau eines multilateralen und freihändlerischen Wirtschaftssystems? Oder konnte ein größeres $\mathrm{Ma} ß$ an Sicherheit nicht doch auf der Basis von Wirtschaftsblöcken erlangt werden, etwa in Form eines britisch-amerikanischen Blocks, auf den dann das System der „Imperial Preferences“ ausgeweitet werden könnte?

In Anknüpfung an den Befund von Leith-Ross, daß sich hinter der Auseinandersetzung mit dem Nationalsozialismus letztlich ein konzeptioneller Gegensatz verbarg und Großbritannien aus deutschlandpolitischen Gründen langfristig dazu gezwungen sein würde, seine protektionistische Wirtschaftspolitik zugunsten der Prinzipien von ökonomischer Sicherheit und internationaler Wirtschaftskooperation aufzugeben, wies Shackle den Gedanken, die Nachkriegsordnung auf der Basis von Wirtschaftsblöcken zu errichten, als friedensgefährdend zurück: „Indeed, it is probably hardly an exaggeration to say that a group solution of post-war problems would give to Germany victory in the economic field, whatever the military outcome. Germany naturally possesses and will always possess an enormous economic pull over her neighbours, due to her importance as an economic and particularly as an industrial unit, whereby her mainly agricul-

93 Vgl. Moggridge, Maynard Keynes, S. 655-663.

${ }^{94}$ FO 371/28900/W 6867, Memorandum R. J. Shackle (Board of Trade), „Economic Reconstruction“, 4.6.1941; DzD I/1, S. 363-372. Dort auch die folgenden Zitate. 
tural neighbours are virtually forced into a position of dependence upon her. This pressure will, of course, be all the stronger if more or less self-contained,economic groups formed in other parts of the world diminish the alternative outlets for European agricultural and industrial products." Wenn aber die Stabilität des Friedens von der Überwindung wirtschaftlicher Autarkiebestrebungen abhänge und der entscheidende Faktor hierbei ein auf der Konvertibilität der Währungen beruhendes monetäres System darstelle, das den ausreichenden Fluß langfristiger Kredite garantiere, so werde der Haltung der USA zwangsläufig eine herausragende Rolle zufallen. Der wirtschaftliche Wiederaufbau, so stellte das Memorandum heraus, beruhe zuvörderst auf der Restauration und Stabilisierung der Währungen sowie auf der Schaffung eines ausreichenden Maßes an internationaler Kaufkraft mittels der Vergabe von Wiederaufbaukrediten. Dazu könnten die USA in dreifacher Weise beitragen: durch finanzielle Hilfe, deren Verteilung über eine internationale Organisation erfolgen solle; durch Importerleichterungen für ausländische Waren, d.h. die Senkung bestehender amerikanischer Zollniveaus, um so den Schuldnerländern die Einnahme von Dollars und die Abtragung ihrer Schulden zu erleichtern; sowie alternativ bzw. ergänzend hierzu durch die Erlassung von Schulden.

Das Herzstück in der Argumentation Shackles war also die Einsicht, daß ein erfolgreicher Wiederaufbau und die Sicherheit vor einem Wiederaufleben deutscher Hegemonialpläne die sinnvolle wirtschaftliche Anbindung Washingtons an den Rekonstruktionsprozeß Europas voraussetzen. Mit anderen Worten, der Gefahr eines kontinentaleuropäischen Blocks unter deutscher Hegemonie entsprach die Gefahr eines amerikanischen Wirtschaftsisolationismus bzw. einer „hemisphärischen Autarkie“ der USA. Und letzterer sollte dadurch vorgebeugt werden, daß die USA zur Übernahme einer ihrer Gläubigerrolle angemessenen Verantwortung für den europäischen Wiederaufbau veranlaßt würden. Hier, so schloß Shackle, lägen denn auch die Einflußmöglichkeiten Großbritanniens. Zwar werde man am Ende des Krieges aufgrund der materiellen Verluste schlechtere Karten in der Hand halten; aber es komme darauf an, die Amerikaner zum richtigen Ausspielen der ihren zu bringen. Aus diesem Grund werde es wohl unvermeidlich sein, weitgehende Zugeständnisse beim Abbau der Empire-Präferenzen zu machen: „An inclination on our part towards Empire self-sufficiency would drive them in this direction [hemispheric self-sufficiency]. On the other hand, a willingness to modify Imperial Preference would probably be the only really effective means we possess of drawing them in the opposite direction, and we know that the present Administration [...] attach great weight to our attitude in this matter as a means of overcoming political obstacles at home."

Shackles Denkschrift stieß auf außerordentlich große Resonanz im Foreign Office. Für den Leiter der Economic Relations Section im Außenministerium, Frank AshtonGwatkin, war sie Ausgangspunkt, den Aufbau des Ministeriums dahingehend zu hinterfragen, ob seine Struktur angesichts der Verzahnung von Wirtschaft und Außenpolitik überhaupt adäquat sei. „Our foreign policy in the future is going to be shaped by political, strategic and economic considerations; all these three fields impinge upon another. " ${ }^{95}$ Hinsichtlich der politischen und strategischen Entscheidungsfaktoren glaubte er zwar das Ministerium, seiner Natur entsprechend, ausreichend arbeitsfähig; aber gerade

${ }^{95}$ FO 371/28900/W6877, Minute Ashton-Gwatkin, 5.6.1941; DzD I/1, S. 372 f. Dort auch die folgenden Zitate. 
Shackles Mahnung, daß von den britisch-amerikanischen Wirtschaftsbeziehungen gleichermaßen die Hoffnungen des Landes auf Frieden wie seine Kriegsangst abhingen, zeige, wie untrennbar Ökonomie und Außenpolitik miteinander verknüpft seien. Dies werfe die Frage auf, ob das Foreign Office in der Lage sei, „to deal effectively with these economic questions, which are going to play a rôle as important as the purely political ones and inextricably mixed up with them". In der Sache selbst bezweifelte AshtonGwatkin Shackles These, daß die expandierenden Märkte des 19. Jahrhunderts, die die Wirtschaftsdynamik am Laufen erhalten hatten, unwiderruflich dahin seien. Es gebe immer noch große „unterentwickelte“ Gebiete, beispielsweise in Osteuropa, der Sowjetunion und China, die noch ausbaufähig seien; zudem werde auch die technisch-industrielle Entwicklung rasant fortschreiten, so daß man nicht von einem Ende des wirtschaftlichen Wachstums und damit der expandierenden Märkte ausgehen könne. In diesem Zusammenhang äußerte Ashton-Gwatkin auch erste Ideen zu einer Teilung Deutschlands. Europa, so kommentierte er, sei wirtschaftlich natürlicherweise in einen schwächeren östlichen Teil, die Eisen- und Kohlereviere Polens, Schlesiens sowie der Tschechoslowakei umfassend, und einen westlichen Teil mit dem Rheinland und Lothringen als Kern geteilt. Bezüglich der Schaffung einer ausreichenden wirtschaftlichen Sicherheit vor Deutschland sei daher zu bedenken, „that in this fact may be found a solution on a natural economic basis for the problem of how to deal with a Germany of overwhelming economic power placed in the centre of Europe, by suggesting a line along which Germany could and should be partitioned, so that Eastern Germany (at least Silesia and East Prussia) should be found in the Eastern, and Western Germany in the Western area".

Sowohl Ronald als auch Makins unterstrichen in ihren Kommentaren die Verpflichtung, die den USA aus ihren enormen Goldvorräten erwachse, wenn nicht andere Staaten ihre Währung aufgrund des Goldmangels abschotten oder in bilaterale Realtauschbeziehungen zurückfallen sollten ${ }^{96}$. John Balfour wies darüber hinaus darauf hin, daß eine ökonomische Nabelschau Großbritanniens Gefahr laufe, die Notwendigkeit eines politischen Konsenses mit den USA auch in deutschlandpolitischen Fragen zu unterschätzen. Der Glaube, alles werde gut, solange sich nur für Großbritannien wirtschaftliche Abhilfen fänden, verkannte nach Balfours Meinung den politischen Kontext. Als oberstes Ziel, dem die britischen Planungen unterzuordnen waren, erblickte er die britisch-amerikanische Zusammenarbeit nach dem Krieg. Die Bereitschaft Washingtons gerade in Wirtschafts- und Währungsfragen dürfe aber nicht einfach vorausgesetzt werden, „unless we, for our part, are ready to go as far as we can in the direction of concessions which will encourage them to undertake the job “97. Nicht zuletzt in der Deutschlandpolitik, wo sich ein Eintreten der USA gegen einen neuerlichen „Diktatfrieden“ abzeichne, gelte daher für die britischen Planungen, „that our ideas about the political future of Europe do not crystallise in a form which would prejudice the prospect of U.S. collaboration in other spheres" ${ }^{\text {"98 }}$. Wie bereits Makins sprach er sich dafür aus, eine Ausdehnung des Systems der Handelspräferenzen auf die Vereinigten Staaten (und Latein-

\footnotetext{
${ }_{96}$ FO 371/28900/W 6887, Minute Ronald, 16.6.1941; Minute Makins, 22.6.1941; beide auch in: DzD I/1, S. $373 \mathrm{f}$.

${ }^{77} \mathrm{FO}$ 371/28900/W 6887, Minute Balfour, 25.6.1941; DzD I/1, S. $374 \mathrm{f}$.

${ }^{98}$ Ebenda.
} 
amerika) ernsthaft zu erwägen. Die intensive Diskussion, die das nationalsozialistische Konzept einer „Neuordnung“ im Herbst des Vorjahres ausgelöst hatte, führte, so wurde deutlich, nicht nur die verschiedenen Linien der britischen Außen-, Wirtschafts- und Sicherheitspolitik der Zwischenkriegszeit, die Keynessche Wirtschaftslehre sowie die unter dem Stichwort „soziale Sicherheit“ im Inneren zu lösenden Problemfelder in einem Schnittpunkt zusammen. Sie zwang zugleich Whitehall zu einer Auseinandersetzung mit der eigenen wirtschaftlichen Entwicklung der zwanziger und dreißiger Jahre. Zwar lagen die Prioritäten des Kriegskabinetts auf den Erfordernissen der Kriegführung, die es den Ministern kaum erlaubten, ihre Aufmerksamkeit möglichen Friedensplanungen zu widmen. Doch auf Beamtenebene war eine Bestandsaufnahme in Gang gesetzt worden, von der jeder Entwurf einer britischen Nachkriegspolitik ihren Ausgang nehmen mußte.

Dies wird besonders deutlich in einem Überblick über die wirtschaftliche Entwicklung der dreißiger Jahre, in dem Ashton-Gwatkin die Rahmenbedingungen einer künftigen Friedensordnung in zehn Lektionen zusammenfaßte. „The first and principal lesson of the nineteen thirties in the politico-economic sphere is", so resümierte er prägnant, "that every symptom of economic nationalism must be regarded as indicating war rather than peace; and that an out-and-out policy of economic nationalism is a high-road towards war." "99 Aus diesem Grund bewertete Ashton-Gwatkin die Schaffung von Handelspräferenzgebieten als friedensbedrohend. Demgegenüber sei ein ausreichendes Maß an Währungsstabilität unabdingbare Voraussetzung für wirtschaftlichen Frieden, der allerdings nicht durch multilaterale Lösungsversuche erreicht werden könne: Nicht nur die Weltwirtschaftskonferenz 1933, sondern gerade auch das trilaterale Finanzabkommen 1936 habe die Unwirksamkeit solcher Verhandlungen demonstriert; der Versuch, Deutschland in ein solches Währungssystem zu integrieren und damit die Aufrüstung des Landes sowie Berlins Offensivpläne einzudämmen, sei de facto in eine Politik des „Economic Appeasement“ umgeschlagen und schließlich durch den Anschluß Österreichs im März 1938 ein für allemal gescheitert ${ }^{100}$. Aufgrund dieses Erfahrungswerts liege die Garantie für stabile Währungen in den Händen Großbritanniens und der USA, deren Beziehungen zueinander daher eine herausragende Bedeutung zukomme. Je weiter sich beide Länder handelspolitisch voneinander entfernten, desto schwieriger sei eine sich ergänzende Währungspolitik zu realisieren; umgekehrt werde ein gemeinsames handelspolitisches Vorgehen auch die Chancen weltweiter Währungsstabilität erhöhen. Auf der Basis einer funktionierenden handels- und währungspolitischen Zusammenarbeit zwischen London und Washington - „but not on any other basis“ - könne dann mit dem Aufbau eines einigermaßen freien internationalen Handelssystems begonnen werden, dem schließlich Frankreich, Deutschland, Italien und Japan gleichberechtigt angehören sollten - „subject to the condition that trade competition must be fair". Würden nämlich diese vier Wirtschaftsmächte ausgeschlossen, so wären sie gezwungen, mit der friedensbedrohenden Schaffung autarker Blöcke zu reagieren. Diese Lektionen, so gab er aber abschließend zu bedenken, ,would seem to endorse the need for the greatest pos-

99 FO 371/28904/W 10371, Memorandum Ashton-Gwatkin, „Notes on Economic Policy in the Nineteen Thirties", 6.8.1941. Dort auch die folgenden Zitate.

${ }^{100}$ Noch im November 1938 hatte der deutsche Botschafter Dirksen ans Auswärtige Amt gemeldet, „daß er [Ashton-Gwatkin] volles Verständnis für unsere wirtschaftlichen Ansprüche auf dem Balkan hat". Dirksen an von Weizsäcker, 9.11.1938, in: ADAP, Serie D, Bd. IV, S. $284 \mathrm{f}$. 
sible freedom in international trade. Complete freedom is impossible since, in each country, the working population [...] must be protected in its employment. There is therefore a fundamental contradiction. [...] The lessons of 1930-39 also show that a healthy international trade depends on agreement for mutual consideration between Great Britain, the United States of America, Germany and France (i.e. the four great import markets); and that such agreement must be preceeded by Anglo-American Agreement on currency and commercial policy."

Die zusammenfassenden Bemerkungen Ashton-Gwatkins, die als Spiegel des Diskussionsstands der drei federführenden Ministerien - Außenministerium, Handelsministerium, Schatzamt - im Herbst 1941 gelten können, lassen ein Grundmuster der britischen Planungen erkennen, in dem deutlich Keynessche Gedanken verarbeitet waren, das zugleich aber auch auf noch ungeklärte Widersprüche hinwies. Hatte Keynes in seiner ersten Reaktion auf die nationalsozialistische „Neuordnung“ die Beibehaltung von Wirtschaftsblöcken noch explizit hervorgehoben und einen Vorteil gegenüber den Plänen des Reichswirtschaftsministeriums ausschließlich in der bona fide-Überlegenheit Londons erblickt, so wich der Gedanke in seinen späteren Ausführungen der Idee einer internationalen Organisation zur Koordinierung der Währungs- und Kreditpolitik und wurde schließlich in den Memoranden des Board of Trade (BoT) und des Foreign Office ausdrücklich zurückgewiesen. Statt dessen wurde die Bedeutung einer britisch-amerikanischen Währungskooperation für eine stabile wirtschaftliche Friedensordnung betont, die ihrerseits die Schaffung multilateraler Handelsbeziehungen ermöglichen sollte. Wie Keynes bereits in diesem frühen Stadium der britischen Kriegszielplanungen verdeutlicht hatte, spielte dabei die Reintegration eines von seiner aggressiven Grundausrichtung befreiten deutschen Industriepotentials eine wichtige Rolle, dessen Austritt aus der internationalen Wirtschaftsgemeinschaft in den dreißiger Jahren, in Ashton-Gwatkins Worten, gesunde Handelsbeziehungen vereitelt hatte. Es begann sich gleichfalls stärker abzuzeichnen, daß der Anspruch auf Vollbeschäftigung als zentrales politisches Anliegen jeder britischen Nachkriegsregierung immer mehr an Gewicht gewann. Ungeklärt war jedoch noch, wie dieser Anspruch und die damit verbundene Forderung Keynes' nach einer interventionistischen Rolle der Regierung mit der Einsicht, daß die Schaffung einer Sicherheit gewährenden Nachkriegsordnung langfristig nur innerhalb eines umfassenden Freihandelssystems realisierbar war, vereinbart werden konnte.

Hier scheint auch ein wichtiger Grund dafür zu liegen, daß London auf die nationalsozialistischen Wirtschaftspläne nicht mit einem gleichwertigen Gegenkonzept, etwa im Sinne eines britischen „New Deal“, antworten konnte. Die internen Diskussionen förderten zunächst ohne Frage den Durchsetzungsprozeß der Keynesschen Wirtschaftstheorien, indem sie sie in engeren Bezug zur Deutschlandpolitik setzten und damit die Erfahrungen mit der Versailler Nachkriegsordnung wiederzubeleben begannen. Zum anderen aber führten sie zu einer Art Bestandsaufnahme der Prämissen der britischen Wirtschafts-, Außen- und Sicherheitspolitik. Als vordringlichste Aufgabe ergab sich für die Planungen Whitehalls zunächst die Lösung des Vermittlungsproblems zwischen einem Nachkriegswirtschaftssystem, das, auf Freihandel und Währungskonvertibilität beruhend, wirtschaftliche Sicherheit in den Mächtebeziehungen versprach, und einer zumindest partiell protektionistischen Wirtschaftspolitik, die den Wiederaufbau der eigenen Industrie begünstigte und soziale Sicherheit nach innen gewährleistete. Dieser Widerspruch ließ die Beamten einen möglichen britisch-amerikanischen Gegensatz antizi- 
pieren, eine Befürchtung, die durch das seit Herbst 1940 aktivere Eingreifen Washingtons und insbesondere durch die Reden Roosevelts und Cordell Hulls Bestätigung zu finden schien. Dennoch wurde die Rolle der USA als voraussichtlich größter Gläubiger nach Kriegsende ausdrücklich anerkannt. Nicht zuletzt um Washington an der Lösung des wirtschafts- und sicherheitspolitischen Problemgeflechts zu beteiligen, kam zukünftigen britisch-amerikanischen Finanzgesprächen eine besondere deutschlandpolitische Bedeutung zu. Hier würde allerdings die britische Regierung zu Zugeständnissen, vor allem im Hinblick auf einen Abbau der Empire-Präferenzen, bereit sein müssen. Dies war mit Blick auf den Wiederaufbau in Europa der Preis, der zu zahlen war, um einen Wirtschaftsisolationismus der USA zu verhindern und Sicherheit vor Deutschland zu schaffen. Schließlich hatten die Diskussionen Fragen nach den ökonomischen Grundlagen der britischen Außenpolitik aufgeworfen, die den machtpolitischen Spielraum Londons sowohl gegenüber Washington, das zum „richtigen“ Ausspielen der Karten bewegt werden sollte, als auch für die Gestaltung der alliierten Deutschlandpolitik absteckten. Mit dieser Bestandsaufnahme war der Anstoß für weitere Kriegszielplanungen gegeben; zugleich waren auch die Problemkreise weitgehend angesprochen, die die Formulierung der britischen Nachkriegspolitik bestimmen würden und von deren Lösung der künftige machtpolitische Standort Großbritanniens abhängen sollte. 